\title{
FINITE GROUP ACTIONS ON THE MODULI SPACE OF SELF-DUAL CONNECTIONS. I
}

\author{
YONG SEUNG CHO
}

\begin{abstract}
Let $M$ be a smooth simply connected closed 4-manifold with positive definite intersection form. Suppose a finite group $G$ acts smoothly on $M$. Let $\pi: E \rightarrow M$ be the instanton number one quaternion line bundle over $M$ with a smooth $G$-action such that $\pi$ is an equivariant map. We first show that there exists a Baire set in the $G$-invariant metrics on $M$ such that the moduli space $\mathscr{M}_{*}^{G}$ of $G$-invariant irreducible self-dual connections is a manifold. By utilizing the $G$-transversality theory of T. Petrie, we then identify cohomology obstructions to globally perturbing the full space $\mathscr{M}_{*}$ of irreducible self-dual connections to a $G$-manifold when $G=\mathbf{Z}_{2}$ and the fixed point set of the $\mathbf{Z}_{2}$ action on $M$ is a nonempty collection of isolated points and Riemann surfaces.
\end{abstract}

\section{INTRODUCTION}

Let $G$ be a finite group, and let $M$ be a simply connected closed smooth 4manifold with a positive definite intersection intersection form and with a smooth action of $G$ on it. Let $\pi: E \rightarrow M$ be a quaternion line bundle with instanton number one and with a $G$-action on $E$ through a bundle isomorphism such that $\pi$ is a $G$-map. The moduli space $\mathscr{M}$ of self-dual connections on $E$ is a $G$-space but may not be a manifold.

To make $\mathscr{M}$ a manifold, Donaldson [9] used a compact perturbation of a Fredholm map, and Freed and Uhlenbeck [14] found generic metrics on $M$. We cannot use these methods directly to make $\mathscr{M}$ a $G$-manifold, because Donaldson's perturbation is not $G$-equivariant and Uhlenbeck's method need not yield a $G$-invariant metric.

We can regard this $G$-action on the bundle as a subgroup of a generalized gauge group [5]. From the $G$-action on the bundle, we can define naturally a $G$-action on the set $\mathscr{C}$ of all connections, the gauge group $\mathfrak{G}, \Omega^{n}\left(\mathscr{G}_{E}\right)$, and $\Omega^{n}(E)$, where $\mathscr{G}_{E}$ is the associated Lie algebra bundle of $E . G$ then acts on $\mathscr{C} / \mathfrak{G}$ and the moduli space $\mathscr{M}$. We use two different methods to transform this mysterious $G$-moduli space $\mathscr{M}$ into a smooth $G$-moduli space with some singularities. In [7] we will find generic metrics on $M$ such that the moduli space $\mathscr{M}$ is a $G$-manifold when $G$ is the group $\mathbf{Z} / 2^{n}$.

Received by the editors January 3, 1989.

1980 Mathematics Subject Classification (1985 Revision). Primary 57N13, 58A14.

Key words and phrases. Group action, self-dual connection, moduli space, generic metric, AtiyahSinger $G$-index, obstruction class, $G$-equivariant perturbation. 
Let $\mathscr{M}^{-G}$ be the set of the $G$-invariant gauge equivalence classes of irreducible self-dual connections. In this paper we first show that

Theorem 4.6. There exists a Baire set in the G-invariant metrics which is obtained by averaging, such that $\mathscr{M}^{-G}$ is a smooth manifold in the moduli $\mathscr{M}^{-}$ of irreducible self-dual connections.

To see the local $G$-structure of $\mathscr{M}$ at each self-dual $G$-invariant connection $\nabla \in \mathscr{M}^{G}$, we will use the Atiyah-Singer $G$-index theorem $[1,3]$ for the $G$ invariant elliptic complex:

$$
0 \rightarrow \Omega^{0}\left(\mathscr{G}_{E}\right) \underset{\delta^{\nabla}}{\stackrel{\nabla}{\rightleftarrows}} \Omega^{1}\left(\mathscr{G}_{E}\right) \stackrel{d^{\nabla}}{\longrightarrow} \Omega_{-}^{2}\left(\mathscr{G}_{E}\right) \rightarrow 0,
$$

where $\delta^{\nabla}$ is the formal adjoint of $\nabla$.

We now assume $G=\mathbf{Z}_{2} \equiv\langle h\rangle$. Suppose that the $G$-fixed point set $F \equiv$ $\left\{P_{i}\right\}_{i=1}^{n_{1}} \cup\left\{T^{\lambda_{i}}\right\}_{i=1}^{n_{2}}$ on $M$, where $P_{i}$ is an isolated fixed point and $T^{\lambda_{i}}$ is a Riemann surface with genus $\lambda_{i}$.

Theorem 3.10. If a connection $\nabla$ is an irreducible (reducible) G-invariant in $\mathscr{M}, h(\nabla)=g(\nabla)$, and $(h g)^{2}=+1$ for some gauge transformation $g$, then we get

$$
\begin{aligned}
& \operatorname{dim} H_{\nabla_{+}}^{1}-\operatorname{dim} H_{\nabla_{+}}^{2}=\frac{1}{4}(10+3 A)(+1), \\
& \operatorname{dim} H_{\nabla_{-}}^{1}-\operatorname{dim} H_{\nabla_{-}}^{2}=\frac{1}{4}(10-3 A),
\end{aligned}
$$

where $A=n_{1}+\sum_{i=1}^{n_{2}} \chi\left(T^{\lambda_{1}}\right)-\operatorname{sign}(h: M)$, sign = signature, and $H_{\nabla_{-}^{+}}^{*}$ means the \pm 1 eigenspace of $h g$.

Theorem 3.10'. If $\nabla$ is a self-dual irreducible connection, $h(\nabla)=g(\nabla)$, and $(h g)^{2}=-1$ for some gauge transformation $g$, then we have

$$
\begin{aligned}
& \operatorname{dim} H_{\nabla_{+}}^{1}-\operatorname{dim} H_{\nabla_{+}}^{2}=\frac{1}{4}(10+A), \\
& \operatorname{dim} H_{\nabla_{-}}^{1}-\operatorname{dim} H_{\nabla_{-}}^{2}=\frac{1}{4}(10-A),
\end{aligned}
$$

where $H_{ \pm}^{*}$ is the \pm 1 eigenspace of $h g$.

Theorem 3.10" . Let $\nabla$ be a self-dual reducible connection and $g(\nabla)=h(\nabla)$ for some gauge transformation $g \notin \Gamma_{\nabla}$. Then we get

$$
\begin{aligned}
& \operatorname{dim} H_{\nabla_{+}}^{1}-\operatorname{dim} H_{\nabla_{+}}^{2}=\frac{1}{4}(14+A), \\
& \operatorname{dim} H_{\nabla_{-}}^{2}-\operatorname{dim} H_{\nabla_{-}}^{2}=\frac{1}{4}(10-A),
\end{aligned}
$$

where $H_{ \pm}^{*}$ is the \pm 1 eigenspace of $g_{1} h g g_{2}$, for some $g_{1}, g_{2} \in \Gamma_{\nabla}$.

By considering the ends of the moduli space [19, 30], together with Theorems 3.10 and $3.10^{\prime}$, we obtain the following theorem. 
Theorem 5.6. The value $A=n_{1}+\sum_{i=1}^{n_{2}}\left(T^{\lambda_{1}}\right)-\operatorname{sign}(h: M)=2$.

We can also get this value for $A$ from the Lefschetz fixed point theorem.

Using this index calculation, we want to perturb the map $\psi: \mathscr{C} / \mathfrak{G} \rightarrow$ $\mathscr{C} \times{ }_{\mathfrak{G}} \Omega^{2}\left(\mathscr{G}_{E}\right)$ given by $\psi(\nabla)=\left(\nabla, R^{\nabla}\right)$ to one transverse to the zero section. This Fredholm $G$-map $\psi$ is locally equivalent to the sum of a $G$-equivariant linear map and a nonlinear $G$-equivariant map with finite dimensional range.

By combining Theorems 3.10 and $3.10^{\prime \prime}$, we then get

Theorem 5.7. Suppose that $\nabla$ is G-invariant, reducible, and self-dual in $\mathscr{M}$. Then there is a G-equivariant perturbation around $\nabla$ in $\beta$ such that the perturbed moduli space has a neighborhood at $\nabla$ which is an open cone on $\mathrm{CP} P^{2}$.

Theorems 3.10 and $3.10^{\prime}$ then yield

Theorem 5.10. If $\nabla$ is $G$-invariant and irreducible in $\mathscr{M}$, then there is a $G$ invariant smooth compact perturbation around $\nabla$ such that the perturbed new moduli space has a smooth 5-dimensional neighborhood at $\nabla$.

We then apply a $G$-transversality technique of Petrie [22] to investigate $G$ transversality on a neighborhood of the fixed point set $\mathscr{M}^{G}$. Consider a fiber bundle $F \rightarrow V \rightarrow X$ where $X=\mathscr{M}^{G}$. Let $X_{0}=\{$ End of $\mathscr{M} \cup$ neighborhood of reducible connections in $\left.\mathscr{M}^{G}\right\} \cap X$, and $F=\operatorname{Hom}_{G}^{S}\left(H_{\nabla_{-}}^{1}, H_{\nabla_{-}}^{2}\right)=$ the surjective $G$-homomorphisms.

Theorem 6.6. (i) To perturb $\psi$ G-transversally throughout a neighborhood of $\mathscr{M}^{G}$ we use the obstruction classes $\Theta_{3}(\psi) \in H^{3}\left(X, X_{0} ; Z\right)$.

(ii) If $\Theta_{3}(\psi)=0$, then the $G$-section $\psi$ has a smooth compact $G$-perturbation $R_{-}+\sigma$ of the self-dual Yang-Mills equations which is transversal to the zero section throughout a small neighborhood of $\mathscr{M}^{G}$.

Let $N\left(\mathscr{M}^{G}\right)$ be a neighborhood of $\mathscr{M}^{G}$ such that $\psi$ is transverse to the zero section throughout $N\left(\mathscr{M}^{G}\right)$. For each $\nabla \in \mathscr{M} \backslash N\left(\mathscr{M}^{G}\right)$, we can choose a local coordinate chart $\Theta_{\nabla \cdot \varepsilon}$ in $\mathscr{C} / \mathfrak{G}$ such that $h\left(\Theta_{\nabla \cdot \varepsilon}\right) \cap \Theta_{\nabla \cdot \varepsilon}=\varnothing$. Let

$$
\begin{aligned}
K=\mathscr{M} \backslash\left\{N\left(\mathscr{M}^{G}\right) \cup \text { End of } \mathscr{M}\right. \\
\cup \text { neighborhood of reducible self-dual connections }\} .
\end{aligned}
$$

The compactness of $K$ and the local splitting of $\psi$ give us a $G$-map $\psi_{1}: \mathscr{C} / \mathfrak{G} \times$ $D^{n}(\eta) \rightarrow \mathscr{C} \times \Omega^{2}\left(\mathscr{G}_{E}\right)$ via $\psi_{1}(x, w)=\psi(x)+\sigma(x, w)$, where $\sigma$ is defined $G$-equivariantly for each $\omega$ in an $\eta$-ball $D^{n}(\eta) \subset R^{n}$ for some $n$.

Theorem 7.6. For almost all $\omega \in D^{n}(\eta)$ the restriction map $\psi()+\sigma(, \omega)$ is transversal to the zero section throughout a neighborhood of $K$.

Thus if the obstruction cohomology classes $\Theta_{3}(\psi)=0$, then we have a smooth $G$-manifold $\mathscr{U}$ of dimension 5 with $\lambda$-singular points each of which has a cone neighborhood on $\mathbf{C} P^{2}$, where $\lambda=\operatorname{rank} H^{2}(M ; Z)$. 


\section{FINITE GROUP ACTIONS ON CONNECTIONS}

Recall that $\mathbf{H} P^{n}$ is the set of 1-dimensional quaternion subspaces in the $(n+1)$-dimensional quaternion space $\mathbf{H}^{n+1}$, and $E=\left\{(l, v) \in \mathbf{H} P^{n} \times \mathbf{H}^{n+1}\right.$ : $v \in l\}$. The projection $P: E \rightarrow \mathbf{H} P^{n}$ given by $P(l, v)=l$ is a natural quaternion line bundle. The associated unit sphere bundle of $E \rightarrow \mathbf{H} P^{n}$ is just the Hopf bundle $S^{4 n+3} \rightarrow \mathbf{H} P^{n}$ which is $4 n$-dimensional classifying of $\mathrm{SU}(2)$-bundles. In case $n=1, \mathbf{H} P^{1}=S^{4}$ and the Hopf bundle $S^{7} \rightarrow S^{4}$ is 4-dimensional classifying of $\mathrm{SU}(2)$-bundles with $C_{2}(E)\left[S^{4}\right]=-1$. We have the following well-known fact:

Theorem 2.1. Let $M$ be a compact oriented 4-manifold. Then there are natural 1-1 correspondences \{equivalence classes of $\mathrm{SU}(2)$-bundles on $M\} \leftrightarrow\left[M^{4}, S^{4}\right]$ $\leftrightarrow H^{4}(M ; \mathbf{Z})=\mathbf{Z}$.

Let $E \rightarrow M^{4}$ be a quaternion line bundle with instanton number one. The instanton number of this line bundle $E$ is defined by $-C_{2}(E)[M]$. If a finite cyclic group $\mathbf{Z} / n \mathbf{Z}$ acts on $M$, we have an induced bundle $h^{*} E \rightarrow M$ where $h$ is a generator of $\mathbf{Z} / n \mathbf{Z}$. Since the bundles $E$ and $h^{*} E$ are bundle isomorphic on $M$ we have a $\mathbf{Z} / n \mathbf{Z}$-action on this bundle $E \rightarrow M$ by composing any bundle isomorphism $E$ to $h^{*} E$ and the induced isomorphism. Similarly if an abelian group acts on $M$, the group acts on the given bundle via pull backs.

Let $G$ be a finite group. Choose Riemannian metrics on the vector bundle $E \rightarrow M$ with respect to which $G$ acts by isometries. Let

$$
\Omega^{k}(E)=\Gamma\left(\Lambda^{k} T^{*} M \otimes E\right)
$$

be the $k$-forms on $M$ with values in $E$. A Riemannian connection on $E$ is a linear map $\nabla: \Omega^{0}(E) \rightarrow \Omega^{1}(E)$ satisfying $\nabla(f \sigma)=d f \otimes \sigma+f \nabla(\sigma)$ and

$$
d\left\langle\sigma_{1}, \sigma_{2}\right\rangle=\left\langle\nabla \sigma_{1}, \sigma_{2}\right\rangle+\left\langle\sigma_{1}, \nabla \sigma_{2}\right\rangle
$$

for any $f \in C^{\infty}(M)$ and any $\sigma, \sigma_{1}, \sigma_{2} \in \Omega^{0}(E)$. We extend a Riemannian connection $\nabla$ on $E$ to the generalized de Rham sequence $\Omega^{0}(E) \stackrel{\nabla}{\longrightarrow}$ $\Omega^{1}(E) \stackrel{d^{\nabla}}{\longrightarrow} \Omega^{2}(E) \rightarrow \cdots$ for any $\theta \otimes \sigma \in \Omega^{1}(E) d^{\nabla}(\theta \otimes \sigma)=d \theta \otimes \sigma-\theta \wedge \nabla \sigma$. The curvature of a connection $\nabla$ is the 2-form $R^{\nabla}=d^{\nabla} \circ \nabla \in \Omega^{2}(\operatorname{Hom}(E, E))$ with values in $\operatorname{Hom}(E, E)$. We have the Bianchi identity $d^{\nabla} R^{\nabla}=0$. The associated Lie algebra bundle $\mathscr{G}_{E}$ of $E$ is given by $\mathscr{G}_{E}=P \times_{\mathrm{SU}(2)} \mathscr{S} \mathscr{U}(2)$, where $P$ is the associated principal bundle of $E$ and $\mathscr{S} \mathscr{U}(2)$ is the Lie algebra of $\mathrm{SU}(2)$. We have the induced metric on $\Lambda^{n} T^{*} M \otimes \mathscr{G}_{E}$ from the metrics on $M$ and $\mathscr{G}_{E}$. The pointwise inner product gives an $L^{2}$-norm in $\Omega^{n}\left(\mathscr{G}_{E}\right)$ by setting $\left(\phi_{1}, \phi_{2}\right)=\int_{M}\left\langle\phi_{1}, \phi_{2}\right\rangle d$ vol for any $\phi_{1}, \phi_{2} \in \Omega^{n}\left(\mathscr{G}_{E}\right)$. The formal adjoint $\delta^{\nabla}: \Omega^{n+1}\left(\mathscr{G}_{E}\right) \rightarrow \Omega^{n}\left(\mathscr{G}_{E}\right)$ of $d^{\nabla}$ is defined by $\left(d^{\nabla} \phi_{1}, \phi_{2}\right)=\left(\phi_{1}, \delta^{\nabla} \phi_{2}\right)$ for all $\phi_{1} \in \Omega^{n}\left(\mathscr{G}_{E}\right)$ and $\phi_{2} \in \Omega^{n+1}\left(\mathscr{G}_{E}\right)$. For each nonnegative integer $l$ we define $\Omega_{l}^{n}\left(\mathscr{G}_{E}\right)$ to be the space of sections whose derivatives of order $\leq l$ are 
square integrable. Thus $\Omega_{l}^{n}\left(\mathscr{G}_{E}\right)$ is the Hilbert space completion of $\Omega^{n}\left(\mathscr{G}_{E}\right)$ with respect to the inner product

$$
\left\|\left(\phi, \phi_{2}\right)\right\|_{l}^{2}=\sum_{i=0}^{l} \int_{M}\left(\nabla^{i} \phi_{1}, \nabla^{i} \phi_{2}\right) d \mathrm{vol} .
$$

Throughout this work we will implicitly use various Sobolev spaces without mention.

Let $\mathscr{C}$ be the space of all $\mathrm{SU}(2)$-connections on $E$. Since the difference of any two connections is in $\Omega^{1}\left(\mathscr{G}_{E}\right), \mathscr{C}$ is an affine space having $\Omega^{1}\left(\mathscr{G}_{E}\right)$ as the vector space of translations. On an oriented Riemannian 4-manifold $M$ there is the Hodge star operator $*: \Lambda^{n} T^{*} M \rightarrow \Lambda^{4-n} T^{*} M$ given by $\alpha \Lambda * \beta=$ $(\alpha, \beta) d$ vol $\in \Lambda^{4} T^{*} M$. On the middle dimension $\Lambda^{2} T^{*} M, *^{2}=1$ and so $\Lambda^{2} T^{*} M=\Lambda_{+}^{2} T^{*} M \oplus \Lambda_{-}^{2} T^{*} M$, where $\Lambda_{ \pm}^{2} T^{*} M$ are the $( \pm 1)$-eigenspaces of $*$.

If we change the metric by multiplying by a positive number $S$, the inner product on the tangent space is multiplied by $S$ and on 2 -forms by $S^{-2}$. However, the volume form is multiplied by $S^{2}$. Thus $*$ is conformally invariant on $\Lambda^{2} T^{*} M$. The adjoint operator $\delta^{\nabla}=-* d^{\nabla} *$ on the 4-manifold. A connection $\nabla$ is (anti) self-dual if $* R^{\nabla}=R^{\nabla}\left(-R^{\nabla}\right.$, respectively).

Let $P \rightarrow M$ be the associated principal bundle of $E$. Let $P \times_{\mathrm{SU}(2)} \mathrm{SU}(2) \rightarrow$ $M$ be the associated Lie group bundle where $\mathrm{SU}(2)$ acts by adjoint on fiber $\mathrm{SU}(2)$. The set of all sections $\mathfrak{G}=\Gamma\left(P \times_{\mathrm{SU}(2)} \mathrm{SU}(2)\right)$ is called the group of gauge transformations. There is a natural action of the gauge group $\mathfrak{G}$ on the space $\mathscr{C}$ of connections, namely $g(\nabla)=g \circ \nabla \circ g^{-1}$ for all $g \in \mathfrak{G}$ and all $\nabla \in \mathscr{C}$. Let $\mathscr{B}=\mathscr{C} / \mathfrak{G}$ and $\mathscr{M}=\mathscr{A} / \mathfrak{G}$, where $\mathscr{A}$ is the set of self-dual connections.

Equivariant self-dual connections were first studied by Fintushel and Stern in [12]. Let a finite group $G$ act on the bundle $E \stackrel{\pi}{\longrightarrow} M$ through bundle isomorphisms such that $\pi$ is a $G$-map. The compatible action induces an action on $\mathscr{C}$. On $\Omega^{0}(E), h(\sigma)=h \circ \sigma \circ h^{-1}$ for any $h \in G$ and any $\sigma \in \Omega^{0}(E)$, where $h^{-1}$ is a diffeomorphism of $M$ and $h$ is a bundle map. For a connection $\nabla$, $h(\nabla)_{v} \sigma=h\left(\nabla_{v}\left(h^{-1} \sigma\right)\right)$, where $\sigma \in \Omega^{0}(E)$ and $v$ is a vector field. There is an action of $G$ on each $\Omega^{k}\left(\mathscr{G}_{E}\right)$ defined by $(h \phi)_{v_{1} \ldots v_{k}}=(h \phi)_{h_{*}^{-1}\left(v_{1}\right) \ldots h_{*}^{-1}\left(v_{k}\right)}$. Since $G$ acts on $M$ by isometries, $G$-action commutes with the *-operation. Also, $G$ acts on the set $\mathscr{A}$ of self-dual connections and $G$-action descends on the moduli space $\mathscr{M}$. The finite group $G$ acts on $\mathscr{C}, \mathscr{B}=\mathscr{C} / \mathfrak{G}$ and $\mathscr{M}=\mathscr{A} / \mathfrak{G}$.

\section{THE INDEX OF THE FUNDAMENTAL ELLIPTIC COMPLEX}

Let $V$ be an $n$-dimensional vector space with inner product $\langle$,$\rangle by defining$ a homomorphism $\Lambda^{2}(V) \rightarrow \operatorname{Hom}(V, V)$ by $(u \wedge v) W=\langle u, w\rangle v-\langle v, w\rangle u$ for all $u, v, w \in V$. We have $\left\langle(u \wedge v) w_{1}, w_{2}\right\rangle+\left\langle w_{1},(u \wedge v) w_{2}\right\rangle=0$. We can identify $\Lambda^{2}(V)$ with the Lie algebra so $(n)$ of the special orthogonal group $\mathrm{SO}(n)$. 
In dimension 4 , the decomposition $\Lambda^{2}=\Lambda_{+}^{2}+\Lambda_{-}^{2}$ corresponds to the decomposition of the Lie algebra $\operatorname{so}(4)=\operatorname{so}(3) \oplus \operatorname{so}(\overline{3})$. So, we can consider $\Lambda_{ \pm}^{2}$ as 3-dimensional Lie algebras. On the Lie group level the homomorphism $\pi: \operatorname{Spin}(4)=\operatorname{Spin}(3) \times \operatorname{Spin}(3) \rightarrow \operatorname{SO}(4)$ defined by $\pi(g, h) x=g x h^{-1}$ has kernel $\{(-1,-1),(1,1)\}$. As a manifold $\operatorname{Spin}(3)=\operatorname{SU}(2)=\operatorname{Sp}(1)=S^{3}$, and $\pi$ is the 2-fold universal covering map. Thus for any oriented Riemannian 4-manifold $M$, we may have, at least locally, the two-complex spinor bundles $V_{+}$(even) and $V_{-}$(odd). Denote the total spin bundle $V=V_{+} \oplus V_{-}$. The complex endomorphism bundle of $V$ is isomorphic to $t^{\prime}$ ' complexified Clifford algebra bundle of the cotangent bundle $T^{*} M$. In particular,

$$
\begin{array}{ll}
\Lambda_{C}^{1}\left(T^{*} M\right) \simeq \operatorname{Hom}_{C}\left(V_{+}, V_{-}\right), & \Lambda_{C}^{3}\left(T^{*} M\right) \simeq \operatorname{Hom}_{C}\left(V_{-}, V_{+}\right), \\
\Lambda_{C_{+}}^{2}\left(T^{*} M\right) \simeq \operatorname{Hom}_{C}\left(V_{+}, V_{+}\right)^{0}, & \Lambda_{C}^{2}\left(T^{*} M\right) \simeq \operatorname{Hom}_{C}\left(V_{-}, V_{-}\right)^{0}, \\
\Lambda_{C}^{0}\left(T^{*} M\right) \simeq \Lambda_{C}^{4} \simeq \Lambda_{C}^{4}\left(T^{*} M\right) \simeq C . &
\end{array}
$$

Here 0 denotes the traceless endomorphisms, and $\Lambda_{C}^{\bullet}$ denotes the complexification of $\Lambda^{\bullet}$.

Let $E \rightarrow M$ be a quaternion line bundle with $k=1$ over a compact oriented simply connected smooth 4-manifold $M$. For a self-dual connection $\nabla \in \mathscr{A}$ there is the fundamental elliptic complex

$$
0 \rightarrow \Omega_{4}^{0}\left(\mathscr{G}_{E}\right) \stackrel{d^{\nabla}}{\longrightarrow} \Omega_{3}^{1}\left(\mathscr{G}_{E}\right) \stackrel{d_{-}^{\nabla}}{\longrightarrow} \Omega_{-2}^{2}\left(\mathscr{G}_{E}\right) \rightarrow 0
$$

where $\Omega_{k}^{\bullet}\left(\mathscr{G}_{E}\right)$ is the Sobolev completion of $\Omega^{\bullet}\left(\mathscr{G}_{E}\right)$ with a Sobolev $k$-norm $\|\phi\|_{k}^{2}=\int_{M}\left\{\|\phi\|^{2}+\cdots+\left\|\nabla^{k} \phi\right\|^{2}\right\} d$ vol.

It is a basic fact that the Sobolev completion of the space of cross sections of a smooth finite-dimensional vector bundle is a Hilbert manifold [19]. The operators $d^{\nabla}$ and $d_{-}^{\nabla}$ in (3.2) are continuous. The gauge group action $\mathfrak{G}$ on the space of connections $\mathscr{C}$ extends to a differentiable action $\mathscr{G}_{4}$ on $\mathscr{C}_{3}$. If we do not complete (3.2) with Sobolev norm, then we cannot guarantee the elliptic operators to be invertible. Moreover the index of (3.2) is independent of the $k$ th Sobolev norm. This fundamental complex was first defined and studied by Atiyah, Hitchin, and Singer [1].

(3.3) The sequence (3.2) is an elliptic complex with finite dimensional cohomologies.

We choose metrics on $E$ and $M$ which are $G$-invariant. Assume that the connection $\nabla$ is $G$-invariant self-dual. Replace the fundamental elliptic complex (3.2) by a single elliptic operator:

$$
\delta^{\nabla}+d_{-}^{\nabla}: \Omega^{1}\left(\mathscr{G}_{E}\right) \rightarrow \Omega^{0}\left(\mathscr{G}_{E}\right) \oplus \Omega_{-}^{2}\left(\mathscr{G}_{E}\right) .
$$

We complexify (3.4) to write this in terms of the Dirac operator associated to the metric:

$$
\delta^{\nabla}+d_{-}^{\nabla}: \Omega_{C}^{1}\left(\mathscr{G}_{C}\right) \rightarrow \Omega_{C}^{0}\left(\mathscr{G}_{C}\right) \oplus \Omega_{C_{-}}^{2}\left(\mathscr{G}_{C}\right),
$$




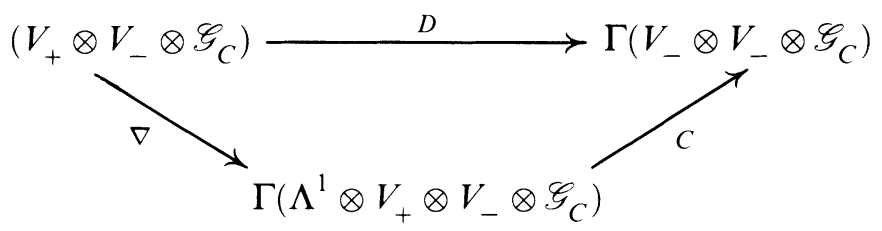

where the connection $\nabla$ on $V_{+} \otimes V_{-} \otimes \mathscr{G}_{f}$ is induced by the Riemannian connection on $V_{+} \otimes V_{-}$and the given self-dual connection on $\mathscr{G}_{C}$, and $C$ is the Clifford multiplication by $T^{*} M$ on $V_{+}$. The two elliptic operators $(3.4)^{\prime}$ and (3.5) have the same index because they have the same symbol and factor through the same connections, that is, they can be written in the form $D_{\phi}=\sum e_{i} \cdot \nabla_{e_{i}} \phi$.

Since we start with the $G$-invariant self-dual connection $\nabla$, the induced Dirac operator $D$ is also $G$-invariant. To compute the $G$-index and $g$-index, for some $g \in G$, we will use the Atiyah-Singer $G$-index theorem.

Theorem 3.6 (Atiyah-Singer $G$-index theorem [24]). Let $G$ be a compact Lie group acting on the compact smooth manifold $M$, and let $D$ be a G-invariant elliptic operator on $M$. Then the g-index of $D$ is related to the fixed point set $M^{g}$ by the formula

$$
\operatorname{Ind}_{g}(D)=(-1)^{m} \frac{\mathrm{Ch}_{g}\left(j^{*} \sigma(D)\right) t d\left(T^{g} \otimes C\right)}{\mathrm{Ch}_{g}\left(\Lambda_{-1} N^{g} \otimes C\right)}\left[T M^{g}\right],
$$

where $m=\operatorname{dim} M^{g}, j: M^{g} \rightarrow M$ is the inclusion map, and $N^{g}$ is the normal bundle of $M^{g}$ in $M$.

Here $m$ will vary from one component to another.

The analytic index, $\operatorname{Ind}_{G}(D)=\operatorname{Ker} D-\operatorname{Coker} D \in R(G)$ is a virtual representation of $G$. For the identity element $e \in G$,

$$
\begin{aligned}
\operatorname{Ind}_{e}(D) & =\operatorname{trace} e: \operatorname{Ind}_{G}(D) \rightarrow \operatorname{Ind}_{G}(D) \\
& =\operatorname{Ch}\left(V_{-} \otimes \mathscr{G}_{C}\right) \operatorname{ch}\left(V_{+}-V_{-}\right) t d(T M \otimes C) e(T M)^{-1}[M] \\
& =\operatorname{ch}\left(V_{-}\right) \cdot \operatorname{ch}\left(\mathscr{G}_{C}\right) \cdot A^{\Lambda}(M) \cdot[M] \\
& =P_{1}\left(\mathscr{G}_{C}\right)[M]+3 \operatorname{ch}\left(V_{-}\right) A^{\Lambda}(M)[M] \\
& =-8 C_{2}(E)[M]+3\left(-b_{0}+b_{1}-b_{2}^{-}\right) \\
& =8 k-\frac{3}{2}(\chi-\tau),
\end{aligned}
$$

where $k=-C_{2}(E)[M], b_{i}=$ the $i$ th Betti number of $M, b_{2}^{-}=$rank of $H_{-}^{2}(M ; C), \chi=$ the Euler characteristic of $M$, and $\tau=$ the signature of $M$. Under our assumption, $k=1$. Since $M$ is simply-connected and the intersection form is positive definite, we have $\chi-\tau=2$.

(3.7) [1]. Let the connection $\nabla$ be $G$-invariant self-dual and let $D$ be the induced Dirac operator. Then $\operatorname{Ind}_{e}(D)=5$, where $e$ is the identity element in $G$. 
Let $G$ act smoothly on $M^{4}$ and preserve the orientation of $M$, where the normal bundle of the fixed point set has even dimensional fibers. Then the fixed point set $M^{G}$ is a disjoint union of even dimensional submanifolds.

Suppose that a $G$-action on the bundle $E \rightarrow M$ has a fixed point set $F=$ $\left\{P_{i}\right\}_{i=1}^{n_{1}} \cup\left\{T^{\lambda_{i}}\right\}_{i=1}^{n_{2}}$ on $M$ where $T^{\lambda_{i}}$ is a Riemannian surface with genus $\lambda_{i}$.

We now specialize to the case that $G=Z_{2}$ and $h$ generates $G$. Let $P \in F$ be an isolated fixed point. Consider the elliptic operator

$$
\delta^{\nabla}+d_{-}^{\nabla}: \Omega^{1}\left(\mathscr{G}_{E}\right) \rightarrow \Omega^{0}\left(\mathscr{G}_{E}\right) \oplus \Omega_{-}^{2}\left(\mathscr{G}_{E}\right) .
$$

To compute $\operatorname{Ind}_{h}\left(\delta^{\nabla}+d_{-}^{\nabla}\right)$ we will use the Atiyah-Singer $G$-index theorem for the Dirac operator (3.5) which has the same index as $\delta^{\nabla}+d_{-}^{\nabla}$.

Fintushel and Stern [12] compute the index of a related elliptic operator. They considered an $\mathrm{SO}(3)$-bundle, and the induced bundles and operators which are all $G$-invariant. We consider an SU(2)-bundle with $G$-action and $G$-invariant elliptic operators. However we consider the whole induced bundle with $G$-action.

If $P \in F$ is an isolated fixed point, then

$$
\left.\operatorname{Ind}_{h}(D)\right|_{[p]}=\frac{\mathrm{Ch}_{h}\left(V_{+}-V_{-}\right) \mathrm{Ch}_{h}\left(V_{-}\right) \mathrm{Ch}_{h}\left(\mathscr{G}_{C}\right) t d\left(T_{p} M \otimes C\right)}{\operatorname{Ch}_{h}\left(\Lambda_{-1} T_{P} M \otimes C\right)}[P] .
$$

Let $\mathscr{G}_{\mathrm{C}}$ be the complexified bundle of the associated Lie algebra bundle $\mathscr{G}_{E}$ of the SU(2)-bundle $E$. The restricted bundle $i^{*} \mathscr{G}_{\mathrm{C}} \rightarrow F$ over the fixed point set $F=M^{G} \subset M$ is an SU(2)-bundle, where $i: F \rightarrow M$ is the inclusion.

Since $B \mathrm{SU}(2)$ is 3-connected, the induced bundles on $F$ are trivial because $F$ has at most two dimensions. The possible actions of $h$ on $i^{*} E \rightarrow F$ are $\left(\begin{array}{ll}1 & 0 \\ 0 & 1\end{array}\right),\left(\begin{array}{cc}-1 & 0 \\ 0 & 1\end{array}\right),\left(\begin{array}{cc}1 & 0 \\ 0 & -1\end{array}\right)$, or $\left(\begin{array}{cc}-1 & 0 \\ 0 & -1\end{array}\right)$ by considering $Z_{2}$-representation on $C^{2}$. However $\left(\begin{array}{cc}-1 & 0 \\ 0 & 1\end{array}\right)$ and $\left(\begin{array}{cc}1 & 0 \\ 0 & -1\end{array}\right)$ do not preserve the SU(2)-structure on $i^{*} E$. The remainders $\left(\begin{array}{ll}1 & 0 \\ 0 & 1\end{array}\right)$ and $\left(\begin{array}{cc}-1 & 0 \\ 0 & -1\end{array}\right)$ act on $\mathrm{SU}(2)$ as the usual multiplication of $\mathrm{SU}(2)$, and on the associated Lie algebra bundle $\mathscr{G}_{E}$ as the adjoint action.

Let

$$
\left(\begin{array}{cc}
i t & a \\
-\bar{a} & -i t
\end{array}\right) \in \mathscr{S} \mathscr{U}(2)
$$

where

$$
\left(\begin{array}{cc} 
\pm 1 & 0 \\
0 & \pm 1
\end{array}\right)\left(\begin{array}{cc}
i t & a \\
-\bar{a} & -i t
\end{array}\right)\left(\begin{array}{cc} 
\pm 1 & 0 \\
0 & \pm 1
\end{array}\right)=\left(\begin{array}{cc}
i t & a \\
-\bar{a} & -i t
\end{array}\right)
$$

So the $G$-action is trivial on $\mathscr{G}_{E} \rightarrow F$ and also on the complexified Lie algebra bundle over $F$. Thus we have

$$
\begin{gathered}
\mathrm{Ch}_{h}\left(\mathscr{G}_{C}\right)=\mathrm{Ch}\left(\mathscr{G}_{C}\right)=3+C_{1}\left(\mathscr{G}_{C}\right)+\cdots=3, \\
t d\left(T M^{h} \otimes C\right)=1+\frac{1}{2} C_{1}\left(T M^{h} \otimes C\right)+\cdots=1,
\end{gathered}
$$


and

$$
\begin{aligned}
& \frac{\mathrm{Ch}_{h}\left(V_{+}-V_{-}\right) \mathrm{Ch}_{h}\left(V_{-}\right)}{\mathrm{Ch}_{h}\left(\Lambda_{-1} T_{p} \otimes C\right)}[P] \\
& \quad=\left[\prod_{i=1}^{2} \frac{\left.e^{\pi i / 2}-e^{-\pi i / 2}\right) e^{-\pi i / 2}}{\left(1-e^{i}\right)\left(1-e^{i}\right)}\right]\left(e^{\pi i}+e^{\pi i}\right)[P]=-\frac{1}{2} .
\end{aligned}
$$

Hence $\left.\left(\operatorname{Ind}_{h} D\right)\right|_{P}=-\frac{3}{2}$, where $\langle h\rangle=Z_{2}$. The contribution to $\operatorname{Ind}_{h}(D)$ on a fixed point component $T^{\lambda_{i}}$, which is a Riemann surface with genus $\lambda_{i}$, is

$$
\begin{aligned}
& \frac{\mathrm{Ch}_{h}\left(V_{+}-V_{-}\right) \mathrm{Ch}_{h}\left(V_{-}\right)}{e\left(T^{h}\right) \mathrm{Ch}_{h}\left(\Lambda_{-1} N^{h} \otimes C\right)}\left[T^{\lambda_{i}}\right] \\
& =\frac{\left(e^{x_{1} / 2}-e^{-x_{1} / 2}\right) e^{-x_{1} / 2}\left(e^{x_{1} / 2+\pi i / 2}-e^{-x_{2} / 2-\pi i / 2}\right) e^{-x_{2} / 2-\pi i / 2}}{X_{1}\left(1-e^{\left(x_{2}+\pi i\right)}\right)\left(1-e^{\left(x_{2}+\pi i\right)}\right)} \\
& \quad \times\left(e^{x_{1}}+e^{\left(e_{2}+\pi i\right)}\right)\left[T^{\lambda_{i}}\right] \\
& =\frac{1}{2}\left(X_{1}\left[T^{\lambda_{i}}\right]-X_{2}\left[T^{\lambda_{i}}\right]\right),
\end{aligned}
$$

where $X_{1}$ and $X_{2}$ represent the Euler classes of the tangent bundle and the normal bundle of $T^{\lambda_{i}}$ respectively. Thus

$$
\left.\operatorname{Ind}_{h}(D)\right|_{T^{\lambda_{i}}}=-\frac{3}{2}\left\{X_{1}\left[T^{\lambda_{i}}\right]-X_{2}\left[T^{\lambda_{i}}\right]\right\}
$$

Theorem 3.8 [3]. Let $X$ be a compact oriented manifold of dimension $4 k$, and let $h$ be an orientation preserving involution with fixed point set $X^{h}$. Let $\left(X^{h}\right)^{2}$ denote the oriented cobordism class of the self-intersection of $X^{h}$ in $X$. Then

$$
\operatorname{sign}(h: X)=\operatorname{sign}\left[\left(X^{h}\right)^{2}\right]
$$

In our case the manifold $M$ has dimension 4 with fixed point set $F=$ $\left\{P_{i}\right\}_{i=1}^{n_{1}} \cup\left\{T^{\lambda_{i}}\right\}_{i=1}^{n_{2}}$. The isolate fixed points have self-intersection 0 . For the Riemann surface $T^{\lambda_{i}}$ the self-intersection of $T^{\lambda_{i}}$ in $M$ is the sum of the signed isolated transverse intersection points. Thus $\operatorname{sign}\left(\left(T^{\lambda_{i}}\right)^{2}\right)=$ the selfintersection of $T^{\lambda_{i}}=\left(X_{2}\right)_{i}\left[T^{\lambda_{i}}\right]$, where $\left(X_{2}\right)_{i}$ is the Euler class of the normal bundle of $T^{\lambda_{i}}$ in $M$. 


$$
\begin{aligned}
\operatorname{sign}(h: M) & =\operatorname{sign}\left(\left(M^{h}\right)^{2}\right) \\
& =\sum_{i=1}^{n_{2}} \operatorname{sign}\left(\left(T^{\lambda_{i}}\right)^{2}\right)=\sum_{i=1}^{n_{2}}\left(X_{2}\right)_{i}\left[T^{\lambda_{i}}\right], \\
\operatorname{Ind}_{h}(D) & =\left.\sum_{i=1}^{n_{1}} \operatorname{Ind}_{h}(D)\right|_{P_{i}}+\left.\sum_{i=1}^{n_{2}} \operatorname{Ind}_{h}(D)\right|_{T^{i_{i}}} \\
& =\sum_{i=1}^{n_{1}}\left(-\frac{3}{2}\right)+\sum_{i=1}^{n_{2}}\left(-\frac{3}{2}\right)\left[\left(X_{1}\right)_{i}\left[T^{\lambda_{i}}\right]-\left(X_{2}\right)_{i}\left[T^{\lambda_{i}}\right]\right] \\
& =-\frac{3}{2}\left\{n_{1}+\sum_{i=1}^{n_{2}} \chi\left(T^{\lambda_{i}}\right)-\sum_{i=1}^{n_{2}}\left(X_{2}\right)_{i}\left[T^{\lambda_{i}}\right]\right\} \\
& =-\frac{3}{2}\left\{n_{1}+\sum_{i=1}^{n_{2}} \chi\left(T^{\lambda_{i}}\right)-\operatorname{sign}(h: M)\right\} .
\end{aligned}
$$

Theorem 3.9. Let $\nabla$ be a G-invariant self-dual connection. Let $D$ be the induced Dirac operator by the fundamental elliptic complex (3.4). Let $F=\left\{P_{i}\right\}_{i=1}^{n_{1}} \cup$ $\left\{T^{\lambda_{i}}\right\}_{i=1}^{n_{2}}$ be the fixed point set on $M$. Then we have

$$
\begin{gathered}
\operatorname{Ind}_{I}(D)=5 \\
\operatorname{Ind}_{h}(D)=-\frac{3}{2}\left\{n_{1}+\sum_{i=1}^{n_{2}} \chi\left(T^{\lambda_{i}}\right)-\operatorname{sign}(h: M)\right\} .
\end{gathered}
$$

If $\nabla$ is a $G$-invariant self-dual connection, we have the $G$-invariant elliptic complex $\delta^{\nabla}+d_{-}^{\nabla}: \Omega^{1}\left(\mathscr{G}_{E}\right) \rightarrow \Omega^{0}\left(\mathscr{G}_{E}\right) \oplus \Omega_{-}^{2}\left(\mathscr{G}_{E}\right)$. By ellipticity this complex has finite dimensional Ker and Coker. The analytic $G$-index of this complex

$$
\begin{aligned}
& =\operatorname{Ker}\left(\delta^{\nabla}+d_{-}^{\nabla}\right)-\operatorname{Coker}\left(\delta^{\nabla}+d_{-}^{\nabla}\right) \\
& =H_{\nabla}^{1}-\left(H_{\nabla}^{0} \oplus H_{\nabla}^{2}\right) \in R(G),
\end{aligned}
$$

where these cohomologies are the cohomologies of (3.2).

The cohomology $H_{\nabla}^{0}=0$ if the connection $\nabla$ is irreducible, otherwise it has dimension one and trivial $G$-action, since $G$ acts on these cohomology groups.

$$
\begin{aligned}
& \left(\operatorname{dim} H_{\nabla_{+}}^{1}+\operatorname{dim} H_{\nabla_{-}}^{1}\right)-\left(\operatorname{dim} H_{\nabla_{+}}^{0}+\operatorname{dim} H_{\nabla_{+}}^{2}+\operatorname{dim} H_{\nabla_{-}}^{2}\right)=5, \\
& \left(\operatorname{dim} H_{\nabla_{+}}^{1}-\operatorname{dim} H_{\nabla_{-}}^{1}\right)-\left(\operatorname{dim} H_{\nabla_{+}}^{0}+\operatorname{dim} H_{\nabla_{+}}^{2}-\operatorname{dim} H_{\nabla_{-}}^{2}\right)=-\frac{3}{2}-A,
\end{aligned}
$$

where $A=n_{1}+\sum_{i=1}^{n_{2}} \chi\left(T^{\lambda_{i}}\right)-\operatorname{sign}(h: M)$ and \pm stands for \pm 1 eigenspace of the generator $h \in G$.

Theorem 3.10. If a connection $\nabla$ is irreducible (reducible) in $\mathscr{M}, h(\nabla)=g(\nabla)$, and $(h g)^{2}=+1$ for some gauge transformation $g$, then

$$
\begin{aligned}
& \operatorname{dim} H_{\nabla_{+}}^{1}-\operatorname{dim} H_{\nabla_{+}}^{2}=\frac{1}{4}(10-3 A), \\
& \operatorname{dim} H_{\nabla_{-}}^{1}-\operatorname{dim} H_{\nabla_{-}}^{2}=\frac{1}{4}(10+3 A),
\end{aligned}
$$

where $H_{\nabla \pm}^{*}$ is the \pm 1 eigenspace of $h g$. 
Note that each element of the fixed point set $\mathscr{M}^{G}$ in the moduli space is a $G$-invariant self-dual connections up to the gauge equivalence because $G$ invariant in $\mathscr{M}$ may not be $G$-invariant in $\mathscr{C}$.

Suppose that $\nabla$ is a self-dual irreducible connection such that $h(\nabla)=g(\nabla)$ for some gauge transformation $g(\neq \pm 1)$ where $\langle h\rangle=G$. Then $(h g) \nabla=\nabla$ and $(h g)^{2} \nabla=\nabla$. We have $(h g)^{2}= \pm 1 \in \mathfrak{G}$. If $(h g)^{2}=I$, then we have the same result as in Theorem (3.9). If $(h g)^{2}=-I$, then $f$ has order 4 on the total space $E$ and $f$ has order 2 on the base manifold $M$, where $f=h g$. Again we have an $f$-invariant fundamental elliptical complex

$$
0 \rightarrow \Omega^{0}\left(\mathscr{G}_{E}\right) \rightarrow \Omega^{1}\left(\mathscr{E}_{E}\right) \rightarrow \Omega_{-}^{2}\left(\mathscr{G}_{E}\right) \rightarrow 0,
$$

As before we have an induced elliptic operator

$$
D:\left(V_{+} \otimes V_{-} \otimes \mathscr{G}_{C}\right) \rightarrow \Gamma\left(V_{-} \otimes V_{-} \otimes \mathscr{G}_{C}\right),
$$

and its index

$$
\begin{aligned}
\operatorname{ind}_{F}(D) & =(-1)^{\operatorname{dim} M^{\prime} / 2} \frac{\mathrm{Ch}_{f}\left(V_{+}-V_{-}\right) \mathrm{Ch}_{f}\left(V_{-}\right) \mathrm{Ch}_{f}\left(\mathscr{G}_{C}\right) t d\left(T M^{f} \otimes C\right)}{e\left(T M^{f}\right) \mathrm{Ch}_{f}\left(\Lambda_{-1} N^{f} \otimes C\right)}\left[M^{f}\right] \\
& =(-1)^{\operatorname{dim} M^{k} / 2} \frac{\mathrm{Ch}_{h}\left(V_{+}-V_{-}\right) \mathrm{Ch}_{h}\left(V_{-}\right) \mathrm{Ch}_{f}\left(\mathscr{G}_{C}\right) t d\left(T M^{h} \otimes C\right)}{e\left(T M^{h}\right) \mathrm{Ch}_{h}\left(\Lambda_{-1} N^{h} \otimes C\right)}\left[M^{h}\right] .
\end{aligned}
$$

The only difference between this formula and the previous formula is that the $h$-Chern character $\mathrm{Ch}_{h}\left(\mathscr{G}_{C}\right)$ is replaced by the $f$-Chern character $\mathrm{Ch}_{f}\left(\mathscr{G}_{C}\right)$. The various associated SU(2)-bundles, especially $\mathscr{G}_{C}$, over the fixed point set $F=M^{f}=M^{h}=\left\{P_{i}\right\}_{i=1}^{n_{1}} \cup\left\{T^{\lambda_{i}}\right\}_{i=1}^{n_{2}}$ on $M$ are trivial. On $E, f$ acts as a multiplication of

$$
\left(\begin{array}{cc}
e^{2 \pi i / 4}, & 0 \\
0, & e^{-2 \pi i / 4}
\end{array}\right)
$$

with order 4. On the associated Lie algebra bundle $\mathscr{G}_{E}, f$ acts adjointly, i.e.,

$$
\left(\begin{array}{cc}
e^{i \theta}, & 0 \\
0, & e^{-i \theta}
\end{array}\right)\left(\begin{array}{cc}
i t, & a \\
-\bar{a}, & -i t
\end{array}\right)\left(\begin{array}{cc}
e^{-i \theta}, & 0 \\
0, & e^{i \theta}
\end{array}\right)=\left(\begin{array}{cc}
i t, & e^{2 i \theta} a \\
-e^{-2 i \theta} a, & -i t
\end{array}\right)
$$

So if we write $\mathscr{G}_{E}=\underline{R} \oplus \underline{C}$, then $f$ acts trivially on $\underline{R}$ and $f$ acts with weight 2 on $\underline{C}$. Using the splitting principle, $\mathrm{Ch}_{f}\left(\mathscr{G}_{C}\right)=e^{x_{1}}+e^{x_{2}} e^{\pi i}+e^{x_{3}} e^{-\pi i}=$ $1-1-1=-1$. Since $\operatorname{td}\left(T M^{h} \otimes C\right)=1$,

$$
\frac{\mathrm{Ch}_{h}\left(V_{+}-V_{-}\right) \mathrm{Ch}_{h}\left(V_{-}\right)}{\mathrm{Ch}_{h}\left(\Lambda_{-1} N^{h} \otimes C\right)}[P]=-\frac{1}{2}
$$

and

$$
\frac{\mathrm{Ch}_{h}\left(V_{+}-V_{-}\right) \mathrm{Ch}_{h}\left(V_{-}\right)}{e\left(T^{\lambda_{i}}\right) \mathrm{Ch}_{h}\left(\Lambda_{-1} N^{h} \otimes C\right)}\left[T^{\lambda_{i}}\right]=\frac{1}{2}\left(\chi\left(T^{\lambda_{i}}\right)-X_{2}\left(T^{\lambda_{i}}\right)\right.
$$


where $X_{2}\left(T^{\lambda_{i}}\right)$ is the self-intersection number of $T^{\lambda_{i}}$. Thus we have

$$
\begin{aligned}
\operatorname{Ind}_{f}(D) & =\sum_{i=1}^{n_{1}}\left[\operatorname{Ind}_{f}(D)\right] P_{i}+\sum_{i=1}^{n_{2}}\left[\operatorname{Ind}_{f}(D)\right]_{T^{i_{i}}} \\
& =\sum_{i=1}^{n_{1}} \frac{1}{2}+\sum_{i=1}^{n_{2}} \frac{1}{2}\left(\chi\left(T^{\lambda_{i}}\right)-X_{2}\left(T^{\lambda-i}\right)\right) \\
& =\frac{1}{2}\left\{\eta_{1}+\sum_{i=1}^{n_{2}} \chi\left(T^{\lambda_{i}}\right)-\operatorname{sign}(h: M)\right\} .
\end{aligned}
$$

Similarly we can calculate $\operatorname{Ind}_{f^{2}}(D)$ and $\operatorname{Ind}_{f^{3}}(D)$.

Theorem 3.9' . Let $\nabla$ be a self-dual irreducible connection, let $h(\nabla)=g(\nabla)$ for some gauge transformation $g,(h g)^{2}=-I$, and let $D$ be the induced elliptic operator by the fundamental elliptic complex (3.4). Let $F=\left\{P_{i}\right\}_{i=1}^{n_{1}} \cup\left\{T^{\lambda_{i}}\right\}_{i=1}^{n_{2}}$ be the G-fixed point set on $M$ and let $f=h g$. Then we have

$$
\begin{aligned}
& \operatorname{Ind}_{f^{0}}(D)=5, \\
& \operatorname{Ind}_{f^{1}}(D)=\frac{1}{2}\left\{\eta_{1}+\sum_{i=1}^{n_{2}} \chi\left(T^{\lambda_{i}}\right)-\operatorname{sign}(h: M)\right\}, \\
& \operatorname{Ind}_{f^{2}}(D)=5, \\
& \operatorname{Ind}_{f^{3}}(D)=\frac{1}{2}\left\{\eta_{1}+\sum_{i=1}^{n_{2}} \chi\left(T^{\lambda_{i}}\right)-\operatorname{sign}(h: M)\right\} .
\end{aligned}
$$

For simplicity let $A \equiv \eta_{1}+\sum_{i=1}^{n_{2}} \chi\left(T^{\lambda_{1}}\right)-\operatorname{sign}(h: M)$.

Now consider the analytic index for the fundamental $f$-invariant elliptic complex: $\operatorname{Ind}_{H}(D)=H_{\nabla}^{1}-H_{\nabla}^{2} \in R(H)$, where $H=\langle f\rangle$. Irreducibly, $H=\langle f\rangle$ decomposition $H_{\nabla}^{1}=\bigoplus_{n=0}^{3} h_{n}^{1} H_{\nabla \cdot n}^{1}, H_{\nabla}^{2}=\bigoplus_{n=0}^{3} h_{n}^{2} H_{\nabla \cdot n}^{2}$, where $h$ acts as $(i)^{n}$ on $H_{\nabla \cdot n}^{*}$ and $h_{n}^{*} \in Z$. Then

$$
\begin{aligned}
& \operatorname{Ind}_{f^{0}}(D)=\left(h_{0}^{1}+h_{1}^{1}+h_{2}^{1}+h_{3}^{1}\right)-\left(h_{0}^{2}+h_{1}^{2}+h_{2}^{2}+h_{3}^{2}\right)=5, \\
& \operatorname{Ind}_{f^{1}}(D)=\left(h_{0}^{1}+i h_{1}^{1}-h_{2}^{1}-i h_{3}^{1}\right)-\left(h_{0}^{2}+i h_{1}^{2}-h_{2}^{2}-i h_{3}^{2}\right)=\frac{1}{2} A, \\
& \operatorname{Ind}_{f^{2}}(D)=\left(h_{0}^{1}-h_{1}^{1}+h_{2}^{1}-h_{3}^{1}\right)-\left(h_{0}^{2}-h_{1}^{2}+h_{2}^{2}-h_{3}^{2}\right)=5, \\
& \operatorname{Ind}_{f^{3}}(D)=\left(h_{0}^{1}-i h_{1}^{1}+i h_{3}^{1}\right)-\left(h_{0}^{2}-i h_{1}^{2}-h_{2}^{2}+i h_{3}^{2}\right)=\frac{1}{2} A .
\end{aligned}
$$

From these we obtain

Theorem 3.10' . Under the hypothesis of Theorem 3.9', we have

$$
h_{0}^{1}-h_{0}^{2}=\frac{1}{4}(10+A), \quad h_{2}^{1}-h_{2}^{2}=\frac{1}{4}(10-A) .
$$

Remark. From the above calculations, $h_{1}^{1}-h_{1}^{2}=0$ and $h_{3}^{1}-h_{3}^{2}=0$. 
Next suppose that $\nabla$ is a self-dual reducible connection such that $h(\nabla)=$ $g(\nabla)$ for some gauge transformation $g \notin \Gamma_{\nabla}$, where $\Gamma_{\nabla}$ is the isotropy subgroup of $\nabla$ which is $\mathrm{SO}(2)$. Then $(h g) \nabla=\nabla$ and $(h g)^{2}(\nabla)=\nabla$. So $(h g)^{2} \in \Gamma_{\nabla}$.

Consider the extended gauge group $\mathfrak{G}^{l}=\{g: E \rightarrow E \mid g$ is a bundle isomorphism which covers id or $h$ on $M\}$. Then we have exact sequences

$$
0 \rightarrow \mathfrak{G} \rightarrow \mathfrak{G}^{l} \rightarrow Z_{2}=\{\text { id }, h\}
$$

and

$$
0 \rightarrow \Gamma_{\nabla} \rightarrow \Gamma_{\nabla}^{\prime} \rightarrow Z_{2} \rightarrow 0
$$

where $\Gamma_{\nabla}^{l}$ is the isotropy subgroup of $\nabla$ in the extended gauge group $\mathfrak{G}^{l}$. Then $\Gamma_{\nabla}^{l}$ is either $\Gamma_{\nabla} \times Z_{2}$ or $O_{2} \simeq \Gamma_{\nabla} \oplus \sigma \Gamma_{\nabla}$ where $\sigma=\left(\begin{array}{cc}-1 & 0 \\ 0 & 1\end{array}\right)$. The extended gauge transformation $h g \in \Gamma_{\nabla}^{l}$ lies on $h$.

If $\Gamma_{\nabla}^{l} \simeq \Gamma_{\nabla} \times Z_{2}$, then $h g=g_{1} h$ for some $g_{1} \in \Gamma_{\nabla}, h g h=g_{1}$. Since $(h g)^{2} \nabla=\nabla,\left(g_{1} g\right) \nabla=\nabla$ and so $g(\nabla)=\nabla$.

This contradicts $g \notin \Gamma_{\nabla}$. Thus $\Gamma_{\nabla}^{l} \not \Gamma_{\nabla} \times Z_{2}$. So if $\Gamma_{\nabla}^{l} \simeq \Gamma_{\nabla} \times Z_{2}$, then we have $g \in \Gamma_{\nabla}$.

If $\Gamma_{\nabla}^{l} \simeq O_{2} \simeq \Gamma_{\nabla} \oplus \sigma \Gamma_{\nabla}$, then $h g=g_{1} \sigma g_{2}$ for some $g_{1}, g_{2} \in \Gamma_{\nabla}$, where $\sigma=g_{1}^{-1} h g g_{2}^{-1}$ covers $h$, and $\nabla$ is a $\sigma$-invariant. From this expression, it is not clear that $\sigma$ has order 2, but by construction $\sigma$ is of the form $\left(\begin{array}{cc}-1 & 0 \\ 0 & 1\end{array}\right)$. So $\sigma$ acts on the Lie algebra bundle $\mathscr{G}_{E}$ as

$$
\left(\begin{array}{cc}
-1 & 0 \\
0 & 1
\end{array}\right)\left(\begin{array}{cc}
i t & a \\
-\bar{a} & -i t
\end{array}\right)\left(\begin{array}{cc}
-1 & 0 \\
0 & 1
\end{array}\right)=\left(\begin{array}{cc}
i t & -a \\
\bar{a} & -i t
\end{array}\right)
$$

Thus we obtain $\mathrm{Ch}_{\sigma}(D)=-1$.

By similar calculations for irreducible connections, we have

Theorem 3.9" . Let $\nabla$ be a self-dual reducible connection, let $h(\nabla)=g(\nabla)$ for some gauge transformation $g \notin \Gamma_{\nabla}$, let $D$ be the induced elliptic operator by the fundamental elliptic complex (3.4), and let $F=\left\{P_{i}\right\}_{i=1}^{n_{1}} \cup\left\{T^{\lambda_{i}}\right\}_{i=1}^{n_{2}}$ be the $G$-fixed point set on $M$. Then $h g \in \Gamma_{\nabla} \oplus \sigma \Gamma_{\nabla}$ where $\sigma \simeq\left(\begin{array}{rr}-1 & 0 \\ 0 & 1\end{array}\right)$. Moreover if $\sigma=g_{1}^{-1} h g g_{2}^{-1}$ for some $g_{1}, g_{2} \in \Gamma_{\nabla}$, then

$$
\operatorname{Ind}_{I}(D)=5, \quad \operatorname{Ind}_{\sigma}(D)=\frac{1}{2} A .
$$

Theorem 3.10". Under the assumption of Theorem 3.9", we have

$$
\begin{aligned}
& \operatorname{dim} H_{\nabla_{+}}^{1}-\operatorname{dim} H_{\nabla_{+}}^{2}=\frac{1}{4}(14+A), \\
& \operatorname{dim} H_{\nabla_{-}}^{1}-\operatorname{dim} H_{\nabla_{-}}^{2}=\frac{1}{4}(10-A) .
\end{aligned}
$$

We will show in Theorem 5.6 that $A=n_{1}+\sum_{i=1}^{n_{2}} \chi\left(T^{\lambda_{i}}\right)-\operatorname{sign}(h: M)=2$.

We can calculate the dimension of the fixed point components of $\mathscr{M}^{G}$ of the moduli space $\mathscr{M}$ by Theorems $3.10,3.10^{\prime}$, and $3.10^{\prime \prime}$. 
Corollary 3.11. Suppose $\nabla \in \mathscr{M}^{G}, h \in G$, and $g \in \mathfrak{G}$.

(i) If $\nabla$ is irreducible and $h(\nabla)=\nabla$, then the dimension of the $\nabla$ component is 1 .

(ii) If $\nabla$ is irreducible, $h(\nabla)=g(\nabla)$, and $(h g)^{2}=-1$, then the dimension of the $\nabla$-component is 3 .

(iii) If $\nabla$ is reducible, then $\nabla$ is a singular cone point of a 1-dimensional fixed point component and a 3-dimensional fixed point component.

\section{Perturbation of $\mathscr{M}^{G}$}

Let $C^{k}=C^{k}(\mathrm{GL}(T M))$ be the set of $C^{k}$-automorphisms of the tangent bundle, that is, the group of gauge transformations for the bundle of frames. Then $C^{k}$ is a Banach manifold [14]. If $g$ is a fixed metric on $M$, then every metric on $M$ is realized by a pull-back metric $\phi^{*}(g)$ of $g$ for some $\phi \in C^{k}$. Since the symmetric group $\operatorname{Sym}(n)=\mathrm{GL}(n) / \mathrm{O}(n)$, many different elements in $C^{k}$ may produce the same metric on $M$. However this does not affect genericity arguments.

Let $P_{-}: \Omega^{2} \rightarrow \Omega_{-}^{2}$ be the projection onto the anti-self-dual 2-forms with respect to the metric $g$. Then $\phi^{*} P_{-} \phi^{-1 *}$ is the projection onto anti-self-dual 2-forms with respect to the metric $\phi^{*}(g)$, that is, the following diagram commutes:

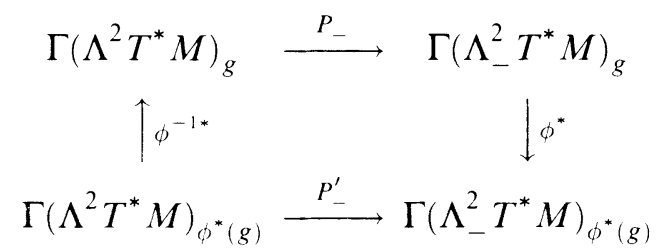

where $P_{-}$is the projection onto the anti-self-dual 2-forms with respect to the metric $\phi^{*}(g)$.

Let $k$ be large enough and define $\Phi: \mathscr{C}_{l-1} \times C^{k} \rightarrow \Omega_{-}^{2}\left(\mathscr{E}_{E}\right)_{l-2}$ by $\Phi(\nabla, \phi)$ $=P_{-}\left(\phi^{-1 *} R^{\nabla}\right)$, where $\mathscr{C}_{l-1}^{-}$is the set of irreducible connections on $E$ with ( $l-1$ )-Sobolev norm. $\Phi(\nabla, \phi)=0$ if and only if $R^{\nabla}$ is self-dual with respect to $\phi^{*}(g)$. Thus $C^{k}$ is chosen as our parameter space so that we can detect selfduality by mapping into a fixed space $\Omega_{-}^{2}\left(\mathscr{G}_{E}\right)_{l-2}$ with respect to the metric $g$.

Lemma 4.1. The map $\Phi: \mathscr{C}_{1-1} \times C^{k} \rightarrow \Omega_{-}^{2}\left(\mathscr{G}_{E}\right)_{l-2}$ is a G-map.

Proof. For any $h \in G$ and any $(\nabla, \phi) \in \mathscr{C}^{\wedge} \times C^{k}$ we have

$$
\begin{aligned}
\Phi(h(\nabla), h(\phi)) & =P_{-}\left[(h(\phi))^{-1 *} R^{h(\nabla)}\right]=P_{-}\left[(h(\phi))^{-1 *} h R^{\nabla} h^{-1}\right] \\
& =P_{-} h\left[(\phi)^{-1 *} R^{\nabla}\right]=h P_{-}\left[\phi^{-1 *} R^{\nabla}\right]=h \Phi(\nabla, \phi) .
\end{aligned}
$$

The fourth equality holds because the metric $g$ is $G$-invariant. Thus we have a $G$-invariant map $\Phi$. 
Corollary 4.2. A connection $\nabla$ is self-dual with respect to $\phi^{*}(g)$ if and only if $h(\nabla)$ is self-dual with respect to $(h \phi)^{*}(g)$.

Theorem 4.3 [14]. The map $\phi$ is smooth and has zero as a regular value.

Since zero is a regular value of $\Phi, \Phi^{-1}(0)$ is an infinite-dimensional Banach manifold of self-dual connections parametrized by the set $C^{k}$ of all metrics. Since the gauge transformation group $\mathfrak{G}_{l}$ acts on $M$ trivially, $\mathfrak{G}_{l}$ acts on $\Phi^{(-1)}(0)$.

Theorem 4.4 [14]. $\Phi^{-1}(0) / \mathfrak{G}_{l} \subset\left(\mathscr{C}_{l-1} / \mathfrak{G}_{l} \times C^{k}\right)$ is a manifold. We have the following diagram:

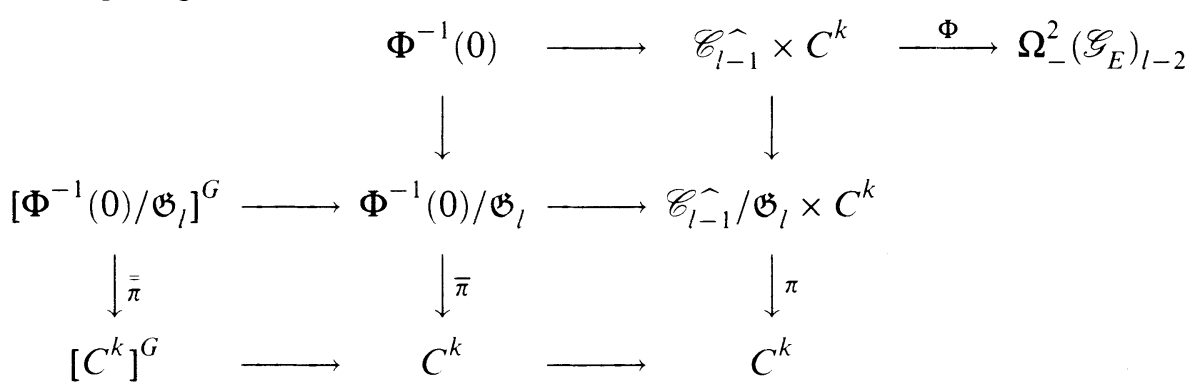

For each metric $\phi \in C^{k}, \pi^{-1}(\phi)=\mathscr{M}_{\phi^{*}(g)}^{\hat{n}}$ is he moduli space of irreducible connections with respect to the metric $\phi^{*}(g)$. As a set, $\Phi^{-1}(0) / \mathfrak{G}_{l}=$ $\bigcup_{\phi \in C^{k}} \mathscr{M}_{\phi^{*}(g)}$.

Theorem 4.5. The manifold $\Phi^{-1}(0) / \mathfrak{G}_{l}$ is a $G$-space.

Proof. Since $\Phi$ is a $G$-map, $\Phi^{-1}(0)$ is a $G$-space. By Corollary 4.2, a connection $\nabla$ is self-dual with respect to $\phi^{*}(g)$ if and only if $h(\nabla)$ is self-dual with respect to the metric $(h \cdot \phi)^{*}(g)$.

For any gauge transformation $g \in \mathfrak{G}_{l}, h \in G, \nabla \in \mathscr{M}_{\phi^{*}(g)}$ we have

$$
\begin{aligned}
h[g(\nabla)] & =h\left[g^{\nabla} g^{-1}\right]=h g^{\nabla} g^{-1} h^{-1} \\
& =\left(h g h^{-1}\right)\left(h^{\nabla} h^{-1}\right)\left(h g^{-1} h^{-1}\right)=h(g) \cdot[h(\nabla)] .
\end{aligned}
$$

Since $G$ acts on $\mathfrak{G}_{l}$ by conjugation, $h(g) \in \mathfrak{G}_{l}$. Hence the map $\mathscr{M}_{\phi^{*}(g)} \stackrel{h}{\longrightarrow}$ $\mathscr{M}_{(h \phi)^{*}(g)}$ given by $[\nabla] \rightarrow[h(\nabla)]$ is well defined and the $G$-action on $\Phi^{-1}(0) / \mathfrak{G}_{l}$ is well defined.

Since the projection map $\pi: \mathscr{C}_{l-1}^{-} / \mathfrak{G}_{l} \times C^{k} \rightarrow C^{k}$ is a $G$-map, the restriction $\bar{\pi}: \Phi^{-1}(0) / \mathfrak{G}_{l} \rightarrow C^{k}$ is also a $G$-map. In [14] it is shown that the map $\bar{\pi}$ is Fredholm and $\bar{\pi}^{-1}(\phi)=\mathscr{M}_{\phi^{*}(g)}$, which has dimension 5 .

The map $\bar{\pi}: \Phi^{-1}(0) / \mathfrak{G}_{l} \rightarrow C^{k}$ is a $G$-Fredholm map. The restriction map $\overline{\bar{\pi}}:\left(\Phi^{-1}(0) / \mathfrak{G}_{l}\right)^{G} \rightarrow\left(C^{k}\right)^{G}$ is a $G$-trivial Fredholm map by Theorem 3.10. By the Sard-Smale Theorem for a Fredholm map between paracompact Banach manifolds we have the following. 
Theorem 4.6. There exists a Baire set of $\left(C^{k}\right)^{G}$ such that $(\overline{\bar{\pi}})^{-1}(\phi)+\left(\mathscr{M}_{\phi^{*}(g)}\right)^{G}$ is a smooth manifold in the moduli space $\mathscr{M}_{\phi^{*}(g)}$ of the irreducible self-dual connections for the metric $\phi^{*}(g)$ on $M$.

We now fix a $G$-invariant metric on $M$ and fix a $G$-invariant metric on the total space $E$ of the bundle such that the fixed point set $\mathscr{M}^{-G}$, in the moduli space $\mathscr{M}^{-}$of the irreducible connections, is a manifold. Note that the above Baire set of $\left(C^{k}\right)^{G}$ is an open dense set for each $k$.

\section{Perturbation in a neighborhood of $\mathscr{M}^{G}$}

In $\S 4$, we showed that for an arbitrary finite group $G$ there is a $G$-invariant generic metric on $M$ such that the fixed point set $\mathscr{M}^{-G}$ in the moduli space $\mathscr{M}^{-}$of irreducible connections is a manifold. We will fix this $G$-invariant metric and set $G=Z_{2}$. In this section we will study local $G$-structures at the fixed points in $\mathscr{M}^{G}$. Then we will locally do $G$-equivariant perturbations at the fixed points. Also we will use the results in $\S 4$ to find necessary conditions under which we can perturb globally in the neighborhood of $\mathscr{M}^{G}$.

Recall the local structure of the moduli space $\mathscr{M}=\mathscr{A} / \mathfrak{G} \subset \mathscr{B}$. Suppose that the fundamental elliptic complex

$$
0 \rightarrow \Omega_{4}^{0}\left(\mathscr{G}_{E}\right) \underset{\delta^{\nabla}}{\stackrel{d^{\nabla}}{\rightleftarrows}} \Omega_{3}^{1}\left(\mathscr{G}_{E}\right) \stackrel{d_{-}^{\nabla}}{\longrightarrow} \Omega_{-2}^{2}\left(\mathscr{G}_{E}\right) \rightarrow 0
$$

has the indicated Sobolev norms, where $\nabla \in \mathscr{M}$. A connection $\nabla$ is reducible iff $\operatorname{dim}_{R}\left(\operatorname{Ker} d^{\nabla}\right)=1$ at $\Omega^{0}\left(\mathscr{G}_{E}\right)$ iff the isotropy group of $\nabla$ is $\Gamma_{\nabla}=\{g \in$ $\left.\mathfrak{G} \mid g^{\nabla} g^{-1}=\nabla\right\}=u(1)$.

Considering the orthogonal decomposition,

$$
T_{\nabla} \mathscr{C}=\Omega_{3}^{1}\left(\mathscr{G}_{E}\right)=\left(\operatorname{Im} d^{\nabla}\right) \oplus\left(\operatorname{Ker} \delta^{\nabla}\right) .
$$

For each $\nabla \in \mathscr{B}$ we have a neighborhood of the form

$$
\begin{cases}\Theta_{\nabla, \varepsilon}=\left\{\nabla+A \mid \delta^{\nabla} A=0,\|A\|_{3}<3\right\} & \text { if } \nabla \text { is irreducible, } \\ \Theta_{\nabla, \varepsilon} / u(1) & \text { if } \nabla \text { is reducible. }\end{cases}
$$

In particular the space $\mathscr{B}^{-}$of irreducible connections is open in $\mathscr{B}$ and is a smooth Hilbert manifold. In the reducible self-dual case $E$ splits as $E=$ $l \oplus \bar{l}$, where $l$ is a complex line bundle on $M$ and the reducible connection $\nabla=\nabla_{1} \oplus \bar{\nabla}_{1}$. Similarly, $\Omega^{n}\left(\mathscr{G}_{E}\right)=\Omega^{n} \oplus \Omega^{n}\left(l^{2}\right)$. Recall that the manifold $M$ is simply connected and has positive definite intersection form. The cohomology groups $H_{\nabla}^{1}$ and $H_{\nabla}^{2}$ of the complex $(*)$ are finite dimensional complex vector spaces, and $H_{\nabla}^{0} \simeq \mathbf{R}$.

For a gauge transformation $g \in \mathfrak{G}$, the anti-self-dual part $R_{-}^{g(\nabla)}=g \circ R_{-}^{\nabla}$ 。 $g^{-1}$. This gives a section of the fibration $\mathscr{F} \equiv \mathscr{C} \times_{\widetilde{\mathfrak{G}}} \Omega_{-}^{2}\left(\mathscr{G}_{E}\right) \rightarrow \mathscr{B}=\mathscr{C} / \widetilde{\mathfrak{G}}$, where $\widetilde{G}=\mathfrak{G} /\{ \pm 1\}$ acts on $\Omega_{-}^{2}\left(\mathscr{G}_{E}\right)$ by adjoint. Namely, the section $\Psi: \mathscr{B}=$ $\mathscr{C} / \widetilde{\mathfrak{G}} \rightarrow \mathscr{C} \times_{\widetilde{\mathfrak{G}}} \Omega_{-}^{2}\left(\mathfrak{G}_{E}\right)$ is given by $\Psi(\nabla)+\left(\nabla, R_{-}^{\nabla}\right)$. 
Let $\nabla \in \mathscr{M}$ be a self-dual connection on $E$. Set $V=\operatorname{Ker} \delta^{\nabla} \subset \Omega_{3}^{1}\left(\mathscr{G}_{E}\right)$ and $W=\Omega_{-2}^{2}\left(\mathscr{G}_{E}\right)$. Define a smooth map $\psi: V \rightarrow W$ be $\psi(A)=d_{-}^{\nabla} A+[A, A]_{-}$. Then the differential $(d \psi)_{0}=d_{-}^{\nabla}: V \rightarrow W$. The map $\psi$ is a Fredholm map. By setting $V_{0}=\operatorname{Ker} d_{-}^{\nabla}$ and $W_{0}=\operatorname{coker} d_{-}^{\nabla}$, we have $d_{-}^{\nabla}: V=V_{0} \oplus V_{1} \rightarrow W=$ $W_{0} \oplus W_{1}$ and the restriction map $d_{-}^{\nabla}: V_{1} \rightarrow W_{1}$ is a Hilbert space isomorphism. Define a map $F: V \rightarrow V$ by $F=\mathrm{id}+\left(d_{-}^{\nabla}\right)^{-1} \circ P_{1} \circ\left(\psi-\left(d^{\nabla}\right)_{0}\right)$, where $P_{1}: W \rightarrow$ $W_{1}$ is the projection. Then $(d F)_{0}=\mathrm{id}$ and $F$ has a local inverse $G$ around 0 . Let $U$ be a small neighborhood of 0 on which $G$ is defined. Define $\Phi: U \rightarrow W_{0}$ by $\phi=P_{0}\left(\psi-d \psi_{0}\right) G$. Then $\Phi(0)=0, d \Phi=0$ and $\Phi$ is commutative with $U(1)$-action, and we have a local commutative diagram:

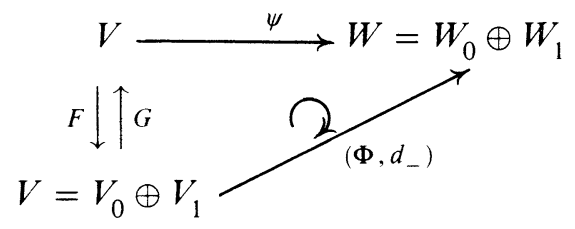

We have local coordinates of the moduli space $\mathscr{M}$ :

$$
\left\{\begin{aligned}
& \mathscr{M} \cap \Theta_{\nabla, \varepsilon} \simeq \Phi^{-1}(0) \text { if } \nabla \text { is irreducible } \\
& \mathscr{M} \cap\left(\Theta_{\nabla, \varepsilon} / U(1)\right) \simeq \Phi^{-1}(0) / U(1) \\
& \text { if } \nabla \text { is reducible. }
\end{aligned}\right.
$$

Let a connection $\nabla$ be a self-dual $G$-invariant connection considering the fundamental elliptic complex

$$
0 \rightarrow \Omega^{0}\left(\mathscr{G}_{E}\right) \underset{\delta^{\nabla}}{\stackrel{d^{\nabla}}{\rightleftarrows}} \Omega^{1}\left(\mathscr{G}_{E}\right) \stackrel{d_{-}^{\nabla}}{\longrightarrow} \Omega_{-}^{2}\left(\mathscr{G}_{E}\right) \rightarrow 0
$$

Lemma 5.3. (i) The covariant derivative $d^{\nabla}: \Omega^{p}\left(\mathscr{G}_{E}\right) \rightarrow \Omega^{p+1}\left(\mathscr{G}_{E}\right)$ is also $G$ invariant.

(ii) The adjoint operator $\delta^{\nabla}$ is G-invariant.

(iii) The map $\psi: V \rightarrow W$ given by $\psi(A)=d_{-}^{\nabla} A+[A, A]_{-}$is G-invariant.

(iv) The map $F: V \rightarrow V$ given by $F=\mathrm{id}+\left(d_{-}^{\nabla}\right)^{-1} p_{1}\left(\psi-d \psi_{0}\right)$ is $G$ invariant.

(v) $\Phi=p_{0}\left[\psi-d \psi_{0}\right] G$ is G-invariant, where the function $G$ is a local inverse of $F$. 
Proof. (i) For any $h \in G, \phi \in \Omega^{p}\left(\mathscr{G}_{E}\right)$, and $v_{0} \cdots v_{p} \in T M$ we have

$$
\begin{aligned}
& \left(d^{\nabla} h \phi\right)_{v_{0} \cdots v_{p}}=\sum_{j=0}^{p}(-1)^{j} \nabla_{v_{i}}\left[(h \phi)\left(v_{0} \cdots v_{j} \cdots v_{p 0}\right)\right] \\
& +\sum_{i<j}(-1)^{i+j}(h \phi)\left(\left[v_{i}, v_{j}\right], v_{0}, \cdots v_{i}^{\wedge} \cdots v_{j} \cdots v_{p}\right) \\
& =\sum_{j=0}^{p}(-1)^{j} \nabla_{v_{j}} h\left(\phi_{\left.h_{*}^{-1} v_{0} \ldots h_{*}^{-1} v_{j} \cdots h_{*}^{-1} v_{p}\right)}\right)
\end{aligned}
$$

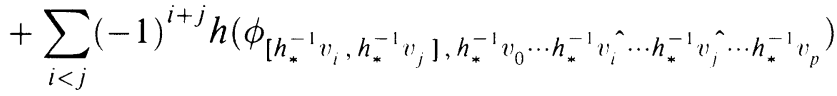

$$
\begin{aligned}
& =h\left(\sum_{j=0}^{p}(-1)^{j} \nabla h_{*}^{-1} v_{j} \phi_{h_{*}^{-1}} v_{0} \cdots h_{*}^{-1} v_{j} \cdots h_{*}^{-1} v_{p}\right. \\
& \left.+\sum_{i<j}(-1)^{i+j} \phi_{\left[h_{*}^{-1} v_{i}, h_{*}^{-1} v_{j}\right], h_{*}^{-1} v_{0} \cdots h_{*}^{-1} v_{i}^{\hat{n}} \cdots h_{*}^{-1} v_{j} \cdots h_{*}^{-1} v_{p}}\right) \\
& =h\left[\left(d^{\nabla} \phi\right)_{h_{*}^{-1} v_{0} \ldots h_{*}^{-1} v_{p}}\right] \\
& =\left[h\left(d^{\nabla} \phi\right)\right]_{v_{0} \cdots v_{p}},
\end{aligned}
$$

where $\nabla_{v} h \cdot \sigma=(\nabla h \sigma)_{v}=(h \nabla \sigma)_{v}=h\left[\nabla_{h^{-1}, v} \sigma\right]$. Thus $d^{\nabla}(h \phi)=h\left(d^{\nabla} \phi\right)$.

(ii) For $\sigma \in \Omega^{0}\left(\mathscr{G}_{E}\right), h \in G$, and $A \in \Omega^{1}\left(\mathscr{G}_{E}\right)$ since $G$ acts isometrically on $E$ and $M$

$$
\begin{aligned}
\left\langle\sigma, \delta^{\nabla}(h A)\right\rangle & =\left\langle d^{\nabla} \sigma, h A\right\rangle=\left\langle h^{-1} d^{\nabla} \sigma, A\right\rangle \\
& =\left\langle d^{\nabla} h^{-1} \sigma, A\right\rangle=\left\langle h^{-1} \sigma, \delta^{\nabla} A\right\rangle=\left\langle\sigma, h \delta^{\nabla} A\right\rangle .
\end{aligned}
$$

For the last three assertions it is sufficient to show that $[A, A]$ is $G$-invariant:

$$
\begin{aligned}
(h[A, A])_{v, w} & =\left(h(A A-A A) h^{-1}\right)_{v, w} \\
& =h A_{h_{*}^{-1},} h^{-1} h A_{h_{*}^{-1} w} h^{-1}-h A_{h_{*}^{-1} w} h^{-1} h A_{h_{*}^{-1},} h^{-1} \\
& =[h(A), h(A)]_{v, w} .
\end{aligned}
$$

Suppose that a connection $\nabla$ is $G$-invariant, reducible, and self-dual. In the fundamental elliptic complex the cohomology groups are $H^{0}=R^{1}, H_{\nabla}^{1} \simeq$ $C^{k+3}$, and $H_{\nabla}^{2} \simeq C^{k}$. They have $G$-actions. Also the isotropy group $\Gamma_{\nabla} \simeq$ $U(1)$ of $\nabla$ in the gauge transformation group $\mathfrak{G}$ acts on the cohomology groups $H_{\nabla}^{1}$ and $H_{\nabla}^{2}$ by scalar multiplication. Of course $H_{\nabla}^{0}$ is a trivial representation of $G$. On the cohomologies $H_{\nabla}^{1}$ and $H_{\nabla}^{2}$ the $G$-action and $\Gamma_{\nabla}$-action are commutative because their representations are linear.

$$
\text { On } H_{\nabla}^{1} \text { and } H_{\nabla}^{2} \text { the } G \text {-action and } \Gamma_{\nabla}=U(1) \text {-action commute. }
$$


Theorem 5.5 [9]. There is an open set $\mathscr{M}_{\lambda_{0}}$ of the moduli space $\mathscr{M}$ of self-dual connections which is a smooth 5-manifold diffeomorphic to $M \times\left(0, \lambda_{0}\right)$ for small $\lambda_{0}>0$ and where complement $K=\mathscr{M} \backslash \mathscr{M}_{\lambda_{0}}$ is compact and $\psi(\nabla) \equiv\left(\nabla, R_{-}^{\nabla}\right)$ is transversal to $\mathscr{M}_{\lambda_{0}}$.

From Theorem 5.5, the end of the moduli space $\mathscr{M}$ is naturally diffeomorphic to $M \times\left(0, \lambda_{0}\right)$ for small $\lambda_{0}>0$, and only contains irreducible self-dual connections. Recall the fixed set $F$ on $M$ is $F=\left\{P_{i}\right\}_{i=1}^{n_{1}} \cup\left\{T^{\lambda_{i}}\right\}_{i=1}^{n_{2}}$ where $T^{\lambda_{i}}$ is a Riemann surface with genus $\lambda_{i}$. The end of the moduli space $\mathscr{M}$ may contain

$$
F \times\left(0, \lambda_{0}\right)=\left\{P_{j} \times\left(0, \lambda_{0}\right)\right\}_{i=1}^{n_{1}} \cup\left\{T^{\lambda_{i}} \times\left(0, \lambda_{0}\right)\right\}_{i=1}^{n_{2}}
$$

as the fixed point components. By Theorem 3.10 some fixed point component in $\mathscr{M}$ has dimension $\frac{1}{4}(10-3 A)$, and by Theorem $3.10^{\prime}$ another fixed component in $\mathscr{M}$ has dimension $\frac{1}{4}(10+A)$, where $A=n_{1}+\sum_{i=1}^{n_{2}}\left(T^{\lambda_{i}}\right)-\operatorname{sign}(h: M)$.

Theorem 5.6. Suppose that a cyclic group $G=\langle h\rangle$ acts on a closed, simply connected 4-manifold $M$ with positive definite intersection form. Let the fixed set $M^{G}=\left\{P_{i}\right\}_{i=1}^{n_{1}} \cup\left\{T^{\lambda_{i}}\right\}_{i=1}^{n_{2}}$ where the $P_{i}$ 's are isolated points and the $T^{\lambda_{i}}$ 's are Riemann surfaces with genus $\lambda_{i}$ respectively. Let $A=n_{1}+\sum_{i=1}^{n_{2}} \chi\left(T^{\lambda_{i}}\right)-$ $\operatorname{sign}(h: M)$. Then $A=2$ if $h$ preserves the orientation, and $A=0$ if $h$ reserves the orientation.

Proof. By the Lefschetz fixed point theorem, the Lefschetz number $L(h)=$ $\chi\left(M^{h}\right)$. Since $M$ is a simply connected closed 4-manifold, $H^{1}(M)=H^{3}(M)=$ 0 . The number $L(h)=2+\operatorname{sign}(h: M)$ if $h$ preserves the orientation, otherwise $L(h)=\operatorname{sign}(h: M)$. Thus we have the desired conclusions.

Theorem 5.7. Suppose that $\nabla$ is G-invariant, reducible, and self-dual in $\mathscr{M}$. Then there is a G-equivariant perturbation around $\nabla$ in $\mathscr{B}$ such that the perturbed moduli space $\mathscr{M}_{1}$ has a neighborhood at $\nabla$ which is an open cone on $\mathrm{C} P^{2}$, where the cone point $\nabla$ is fixed by $G$.

Proof. By Lemma 5.3 the differential map $\psi: V \equiv \operatorname{Ker}\left(\delta^{\nabla}\right) \subset \Omega^{1}\left(\mathscr{G}_{E}\right) \rightarrow W=$ $\Omega_{-}^{2}\left(\mathscr{G}_{E}\right)$ given by $\psi(A)=d_{-}^{\nabla} A+[A, A]_{-}$decomposes as a map $\left(\Phi, d_{-}^{\nabla}\right): H^{1} \oplus$ $V_{1} \rightarrow H^{2} \oplus W_{1}$ by a diffeomorphic $G$-invariant. The restriction map $\left.d_{-}^{\nabla}\right|_{V_{1}}$ is a Hilbert space isomorphism and $\Phi, d_{-}^{\nabla}$ are $G$-invariant. By Theorem 3.10 and Theorem 5.6

$$
\operatorname{dim} H_{\nabla+}^{1}-\operatorname{dim} H_{\nabla+}^{2}=2, \quad \operatorname{dim} H_{\nabla_{-}}^{1}-\operatorname{dim} H_{\nabla-}^{2}=4 .
$$

If $h(\nabla)=g(\nabla)$ for some gauge transformation $g \notin \Gamma_{\nabla}$, then by Theorem $3.10^{\prime \prime}$

$$
\operatorname{dim} H_{\nabla+}^{1}-\operatorname{dim} H_{\nabla_{+}}^{2}=4, \quad \operatorname{dim} H_{\nabla_{-}}^{1}-\operatorname{dim} H_{\nabla_{-}}^{2}=2 .
$$

The map $\psi$ is a $G$-equivariant submersion if and only if the map $\Phi$ is a $G$ equivariant submersion. We can easily perturb $\Phi$ into a $G$-equivariant submersion. For example, in the first case, by Schur's Lemma the map $\Phi$ decomposes 
as

$$
\begin{aligned}
\Phi: H_{\nabla}^{1} & =H_{\nabla+}^{1} \oplus H_{\nabla-}^{1}=C^{k_{1}+1} \oplus C^{k_{2}+2} \rightarrow H_{\nabla}^{2} \\
& =H_{\nabla+}^{2} \oplus H_{\nabla-}^{2}=C^{k_{1}} \oplus C^{k_{2}} .
\end{aligned}
$$

From this decomposition we can choose a map $h: H_{\nabla}^{1} \rightarrow H_{\nabla}^{2}$ which is linear surjective and $G$-invariant. We choose a smooth cutoff function $\rho \in C_{0}\left(\Theta_{\nabla \cdot \varepsilon}\right)$ such that $\rho \equiv 1$ near 0 . Then $\Phi+\rho(h-\Phi): H_{\nabla}^{1} \rightarrow H_{\nabla}^{2}$ has a $C$-linear surjective derivative $h$ at zero. By (5.2) the new zero set modulo $\Gamma_{\nabla}$ is a cone on $\mathbf{C} P^{2}$.

Suppose that a reducible self-dual connection $\nabla$ is not $G$-invariant. We choose an open neighborhood $\Theta_{\nabla \cdot \varepsilon} / U(1)$ in $\mathscr{B}$ such that $\left(\Theta_{\nabla \cdot \varepsilon} / U(1)\right) \cap$ $h\left(\Theta_{\nabla \cdot \varepsilon} / U(1)\right)=\varnothing$. We will show that the connection $h(\nabla)$ is also self-dual reducible. Since $h\left(\delta^{\nabla} A\right)=\delta^{h(\nabla)}(h(A))$ we have a map $h: \operatorname{Ker} \delta^{\nabla} \rightarrow \operatorname{Ker}\left(\delta^{h(\nabla)}\right)$, where

$$
h(\psi(A))=h\left[d_{-}^{\nabla} A+[A, A]_{-}\right]=d_{-}^{h(\nabla)}(h A)+[h A, h A]_{-} .
$$

Thus we have a commutative diagram:

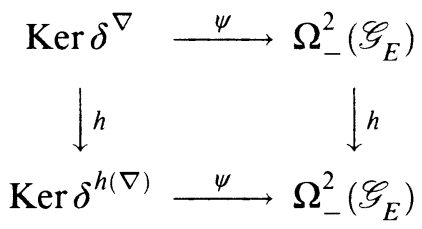

By the Kuranishi technique, $\psi \simeq\left(\phi, d_{-}^{\nabla}\right): H_{\nabla}^{1} \oplus V_{1} \rightarrow H_{\nabla}^{2} \oplus W_{1}$ where the restriction $\phi: H_{\nabla}^{1} \rightarrow H_{\nabla}^{2}$ is a $\Gamma_{\nabla}$-map. The action of the isotropy group $h: \Gamma_{\nabla} \rightarrow \Gamma_{h(\nabla)}$ is a diffeomorphism. After a compact perturbation we have

(5.8) $h:$ [cone on $\mathbf{C} P^{2}$ at $\left.[\nabla]\right] \rightarrow\left[\right.$ cone on $\mathbf{C} P^{2}$ at $\left.h(\nabla)\right]$ is a diffeomorphism except at the cone point $[\nabla]$.

We set $\lambda=\frac{1}{2} \#\left\{u \in H^{2}(M: Z) \mid u \cdot u=1\right\}$. We have $\lambda$-reducible self-dual gauge equivalence classes $\left[\nabla^{1}\right], \ldots,\left[\nabla^{\lambda}\right]$. There is a compact perturbation $\psi_{1}=\psi+\sigma$ such that $\psi_{1}=\psi$ outside small cone-neighborhoods of the $\nabla^{i}$ 's. The differential $d \psi_{1}$ is also a Fredholm operator which has the same index as $d \psi$.

Next we would like to perturb the new moduli space $\mathscr{M}_{1}=\left\{\nabla \in \mathscr{B}: \psi_{1}(\nabla)=\right.$ $0\}$, where $\psi_{1}=\psi+\sigma: \mathscr{B} \rightarrow \mathscr{C} \times \mathfrak{G} \Omega_{-}^{2}\left(\mathscr{G}_{E}\right), G$-equivariantly to a smooth 5manifold with $\lambda$-singularities, where each singularity is a cone neighborhood on $\mathrm{C} P^{2}$.

We have the smooth part $\mathscr{M}_{\lambda_{0}} \cup \mathscr{M}^{\wedge} \cup$ \{open cone on $\mathbf{C} P^{2}$ at each reducible connections $\}$ in the moduli space $\mathscr{M}_{1} \subset \mathscr{B}$ with $\lambda$-singularities. We would like to perturb a small neighborhood of $\mathscr{M}^{-G}$ first locally and then globally by using the Petrie $G$-transversality argument. 
Suppose a connection $\nabla$ is $G$-invariant, (self-dual) irreducible and $\psi_{1}(\nabla)=$ 0 . Locally the map $\psi_{1}: V=\operatorname{Ker} \delta^{\nabla} \rightarrow W=\Omega_{-}^{2}\left(\mathscr{G}_{E}\right)$ is a Fredholm operator, $\left(d \psi_{1}\right)_{0}: V \rightarrow W$ has index 5 with splitting $V=\operatorname{Ker}\left(d \psi_{1}\right)_{0} \oplus V_{1}, W=$ $\operatorname{coker}\left(d \psi_{1}\right)_{0} \oplus W_{1}$, and $\operatorname{Ker}(d \psi)_{0}=R^{k+5}, \operatorname{coker}\left(d \psi_{1}\right)_{0}=R^{k}$. The restriction map $\left(d \psi_{1}\right)_{0} l_{v}$ is a Hilbert isomorphism.

To see the local structure at irreducible connection $\nabla$ we would like to use the Kuranishi argument for this Fredholm map $\psi_{1}: V \rightarrow W$. Define a differentiable map $F=\mathrm{id}+\left(d \psi_{1}\right)^{-1} \circ p_{1} \circ\left(\psi_{1}-d \psi_{1}\right): V \rightarrow V$ where $p_{1}: W \rightarrow W$ is the orthogonal projection. Then $d F=$ id. So $F$ is diffeomorphic in a neighborhood of $F^{-1}(0)$. Define a map $Q: \operatorname{Ker}\left(d \psi_{1}\right)_{0} \rightarrow \operatorname{coker}\left(d \psi_{1}\right)_{0}$ by $Q=p_{0} \circ \psi \circ F^{-1}$ around the zero, where $p_{0}: W \rightarrow \operatorname{coker}\left(d \psi_{1}\right)_{0}$ is the orthogonal projection. By Lemma 5.3 these maps are all $G$-equivariant. So the map

$$
\left(Q,\left(d \psi_{1}\right)_{0}\right): \operatorname{Ker}\left(d \psi_{1}\right)_{0} \oplus V_{1} \rightarrow \operatorname{coker}\left(d \psi_{1}\right)_{0} \oplus W_{1}
$$

is smooth $G$-equivariant and $\psi_{1}=\left(Q,\left(d \psi_{1}\right)_{0}\right) \circ F$ is $G$-equivariant decomposition. We would like to perturb the map

$$
Q: \operatorname{Ker}\left(d \psi_{1}\right)_{0} \rightarrow \operatorname{coker}\left(d \psi_{1}\right)_{0}
$$

to be a map whose derivative is surjective and $G$-equivariant.

For $\nabla \in \mathscr{M}^{\neg G}, h(\nabla)=g(\nabla)$. If $(h g)^{2}=1$, then by Theorem 3.10, since $A=2$,

$$
\operatorname{dim} H_{\nabla_{+}}^{1}-\operatorname{dim} H_{\nabla_{+}}^{2}=1, \quad \operatorname{dim} H_{\nabla_{-}}^{1}-\operatorname{dim} H_{\nabla_{-}}^{2}=4 .
$$

If $(h g)^{2}=-1$, then by $\left(3.10^{\prime}\right)$ we have

$$
\operatorname{dim} H_{\nabla_{+}}^{1}-\operatorname{dim} H_{\nabla_{+}}^{2}=3, \quad \operatorname{dim} H_{\nabla_{-}}^{1}-\operatorname{dim} H_{\nabla_{-}}^{2}=2 .
$$

The map $\psi_{1}$ is a $G$-equivariant submersion if and only if the map $Q$ is a $G$ equivariant submersion. In general $Q$ is not a submersion. By Shur's Lemma, the $G$-equivariant map $Q$ splits as follows:

$$
\left\{\begin{aligned}
\text { (i) If }(h g)^{2} & =1, \text { then } \\
Q: H_{\nabla}^{1} & =R^{k+5}=\left(R^{k_{1}+1}\right)_{+} \oplus\left(R^{k_{2}+4}\right)_{-} \rightarrow H_{\nabla}^{2} \\
& =R^{k}=R_{+}^{k_{1}} \oplus R_{-}^{k_{2}}, \\
\text { (ii) If }(h g)^{2} & =-1, \text { then } \\
Q: H_{\nabla}^{1} & =R^{k+5}=\left(R^{k_{1}+3}\right)_{+} \oplus\left(R^{k_{2}+2}\right)_{-} \rightarrow H_{\nabla}^{2} \\
& =R^{k}=R_{+}^{k_{1}} \oplus R_{-}^{k} .
\end{aligned}\right.
$$

From this splitting we can easily choose a map $h: R^{k+5} \rightarrow R^{k}$ which is a $G$-invariant epimorphism. Choose a smooth cutoff function $\rho \in C_{0}\left(\Theta_{\nabla \cdot \varepsilon}\right)$ with $\rho \equiv 1$ near 0 . Then the map $(1-\rho) Q+\rho h: R^{k+5} \rightarrow R^{k}$ is $G$-equivariant and its derivative is an epimorphism near 0 . 
Theorem 5.10. If a connection $\nabla$ is G-invariant, self-dual, and irreducible in $\mathscr{M}$ then there is a G-invariant smooth compact perturbation around $\nabla$ such that the perturbed new moduli space has a smooth 5-dimensional neighborhood at $\nabla$.

Proof. By the above construction and replacing $\psi_{1}$ by $\left[(1-\rho) Q+\rho h,\left(d \psi_{1}\right)_{0}\right]$, we have the result.

We have shown that we can locally perturb each $G$-invariant self-dual connection into a $G$-invariant manifold. We now would like to find conditions under which we can perturb a neighborhood of the fixed point set $\mathscr{M}^{G}$ into a $G$-equivariant smooth neighborhood of $\mathscr{M}^{G}$. To do tr , we introduce Petrie's $G$-transversality argument and then apply it to our case.

The $G$-transversality argument gives a solution in terms of an obstruction theory and by giving a criterion for the vanishing of the obstructions.

\section{OBSTRUCTIONS FOR $G$-TRANSVERSALITy}

We would like to introduce two basic ideas. First, the problem of equivariant transversality is a global phenomena whereas the nonequivariant situation is local. Second, Shur's Lemma applied to the equivariant vector bundles involved along with transversality gives a splitting of the problems into two parts.

The fixed point part was already done by using generic metrics on $M$. So our main interest is the transversality obstruction.

More precisely, suppose that three smooth $G$-manifolds $N, M$ and $Y$ are given, with $Y \subset M$ a $G$-invariant submanifold, and a proper $G$-map $f: N \rightarrow M$ which is transverse to $Y$ with $X=f^{-1}(Y)$ and $H \subseteq G$. Then $f^{H}$ is transverse to $Y^{H} \subset M^{H}$ and the normal bundle $\nu(X, N)$ of $X$ in $N$ has a splitting $\nu(X, N)^{H} \oplus \nu(X, N)_{H}$ with $\nu(X, N)^{H}=\nu\left(X^{H}, N^{H}\right)$ and $\nu\left(X^{H}, N\right)=\left.\nu(X, N)_{H}\right|_{X^{H}} \oplus \nu\left(X^{H}, X\right)$. The fact that $f$ is transverse to $Y$ throughout $X^{H}$ is expressed by the following two equations:

$$
\begin{aligned}
& \nu\left(X^{H}, N^{H}\right)=\left(f^{H}\right)^{*} \nu\left(Y^{H}, M^{H}\right), \\
& \left.\nu\left(N^{H}, N\right)\right|_{X^{H}}=\left(f^{H}\right)^{*} \nu(Y, M)_{H} .
\end{aligned}
$$

By Shur's Lemma, equation (1) depends only on $f^{H}: N^{H} \rightarrow M^{H}$ and is concerned with the action of the normalizer of $H \bmod H$ on $N^{H}$ and $M^{H}$, which by induction can be assumed to act freely. Since there is no $H$-action the problem of $f^{H}$ being transverse to $Y^{H}$ in $M^{H}$ is treated by Thom transversality and in particular gives $X^{H}=\left(f^{H}\right)^{-1}\left(Y^{H}\right)$ as a submanifold of $N$. It is equation (2) which provides the basis for the transversality obstruction theory. Define the $G$-fiber bundle $V_{\xi, \eta}=\operatorname{Hom}^{s}(\xi, \eta)$ of real surjective homomorphisms of the $G$-vector bundle $\xi$ over $Y$ onto the $G$-vector bundle $\eta$ over $Y$. The action of $G$ is defined by conjugation on $V_{\xi, \eta}$. Then $V_{\xi, \eta}^{H}$ is a $G / H$ fiber bundle over $Y^{H}$ if $H$ is normal in $G$. The fiber over $y \in Y^{H}$ is $V(H)_{y} \equiv \operatorname{Hom}_{H}^{s}\left(\xi_{y}, \eta_{y}\right)$, the space of real surjective $H$-homomorphisms from the fiber $\xi_{y}$ to $\eta_{y}$. Then Petrie shows the following theorem holds. 
Theorem 6.1 ( $G$-transversality theorem [22]). Let $f: N \rightarrow M$ be transverse to $Y$ on $Z_{h-1}=\bigcup_{k>h} N^{k}$, and without loss of generality suppose $f^{H} \pitchfork Y^{H}$. Let $X^{k}=\left(f^{k}\right)^{-1}\left(Y^{k}\right), k \geq H$, and $X_{H}=\bigcup_{k>H} X^{k}$. Then there is a G-invariant neighborhood $W$ of $Z_{h-1}$ and a proper G-homotopy of $f$ rel $W \cup Z_{H}$ to a map $Q \pitchfork Y$ on $Z_{H}$ iff a sequence of obstructions

$$
O_{n}(f, K) \in H^{n}\left(X^{H} / N(H), X_{H} / N(H), \pi_{n-1} V(H)\right)
$$

vanishes. Here $V(H)$ is a function of the components of $X^{H}$. The value of $V(H)$ at a component $P$ of $X^{H}$ is

$$
V(K)_{x}=\operatorname{Hom}_{H}^{s}\left(\nu\left(H^{H}, N\right)_{x}, \nu(Y, M)_{H, f(x)}\right)
$$

for $x \in P \subset X^{H}$.

Moreover let $\widehat{H}$ be the set of irreducible representations of $G$,

$$
\nu\left(N^{H}, N\right)_{\chi}=\sum_{\chi \in \widehat{H}} a_{\chi} \chi, \quad \nu(Y, M)_{H, f(x)}=\sum_{\chi \in \widehat{H}} b_{\chi} \chi,
$$

where $a_{\chi}$ and $b_{\chi}$ are integers, $x \in P . D_{\chi}=\operatorname{Hom}_{H}(\chi, \chi)$ is a division algebra, $\operatorname{dim} D_{\chi} \equiv d_{\chi}$. Then $V(H)_{x}=\prod_{\chi \in \widehat{H}} \mathrm{GL}\left(a_{\chi}, D_{\chi}\right) / \mathrm{GL}\left(a_{\chi}-b_{\chi}, D_{\chi}\right)$.

Remarks. (i) $\operatorname{dim} X^{H}=0$ or $\operatorname{dim} Y^{H}-\operatorname{dim} M^{H}+\operatorname{dim} N^{H}$.

(ii) The cohomology obstruction classes $O_{*}(f, K)$ should be understood in two ways:

1. as components of $X^{H}$ : if $X^{H}=\bigcup_{1}^{n} X_{j}^{H}, x_{j} \in X_{j}^{H}$, and $X_{H}^{j}=X_{H} \cap X_{j}$, then

$$
\begin{aligned}
O_{*} & (f, H) \\
= & \prod_{j=1}^{n} O_{*}(f, H)_{j} \in \prod_{j=1}^{n} H^{*}\left(X_{j}^{H} / N(H), X_{H}^{j} / N(H), \pi_{*-1} V(H)_{x_{j}}\right)
\end{aligned}
$$

2. as representations of $G$ :

$$
\begin{gathered}
O_{*}(f, H)=\prod_{\chi \in \widehat{H}} O_{*}(f)_{\chi}, \\
O_{*}(f)_{\chi} \in H^{*}\left(X^{H} / N(H), X_{H} / N(H), \pi_{*-1} V(H)^{\chi}\right) .
\end{gathered}
$$

(iii) If

$$
\operatorname{dim} X^{H} \leq \min _{\substack{\chi \in \widehat{H} \\ b_{\chi} \neq 0}}\left\{d_{\chi}\left(a_{\chi}-b_{\chi}+1\right)-1\right\},
$$

then the obstruction $O_{n}(H, f)=0$ for all $n$.

Recall that $\psi$ is a cross section of fibration

$$
\mathscr{F}=\mathscr{C} \times_{\mathfrak{G}} \Omega_{-}^{2}\left(\mathscr{G}_{E}\right) \rightarrow \mathscr{B}=\mathscr{C} / \mathfrak{G}
$$


which is a smooth vector bundle associated to the principal bundle $\mathscr{C}^{\wedge} \rightarrow \mathscr{B}^{\text {个. }}$. Let $Z$ be the zero section $\mathscr{M}_{1}=\left\{\nabla \in \mathscr{B} \mid \psi_{1}(\nabla)=0\right\}$. Then $Z$ is the moduli space of the perturbed connections, which is also perturbed at the reducible self-dual connections.

Let $X=\mathscr{M}_{1}^{G}$ and $X_{0}=\left\{\mathscr{M}_{\lambda_{0}} \cup\right.$ open cone neighborhoods at each self-dual connections $\} \cup X$. Then $X \backslash X_{0}$ is compact.

We now apply Theorem 6.1. In our case $H=G=Z_{2}, Z_{h-1}=\varnothing, X_{H}=\varnothing$, $X=X^{H}$, and, by the construction of $\mathscr{M}_{1}^{G}$, the map $\psi_{1}: \mathscr{B} \rightarrow \mathscr{F}$ has a restriction $\psi_{1}^{H}$ such that $\psi_{1}^{H} \cap Z^{H}$ throughout $X$. Let us consider the obstruction classes $O_{n}\left(\psi_{1}\right) \in H^{n}\left(X, X_{0}: \pi_{n-1}(V(H))\right)$ where $V(H)$ is a fiber bundle over $X$. The fiber over $x \in X$ is $V(H)_{x}=\operatorname{Hom}_{H}^{s}\left(\nu\left(\mathscr{B}^{H}, \mathscr{B}\right)_{x} \nu(Z, F)_{H, x}\right)$ where $x$ is an irreducible $G$-invariant self-dual connection. From the local structure at $x=\nabla, T_{\nabla} \mathscr{B}=\operatorname{Ker} \delta^{\nabla}=R^{k+5} \oplus V_{1}$ for some $k$. By (5.9) the map $\psi_{1}: R^{k+5} \oplus V_{1} \rightarrow R^{k} \oplus W_{1}$ is split as follows. For any $\nabla \in X \backslash X_{0}$ and $h(\nabla)-g(\nabla)$, if $(h g)^{2}=+1$, then

$$
\psi_{1}=\left(Q, d_{-}\right):\left(R_{+}^{k_{1}+1} \oplus R_{-}^{k_{2}+4}\right) \oplus V_{1} \rightarrow\left(R_{+}^{k_{1}} \oplus R_{-}^{k_{2}}\right) \oplus W_{1},
$$

if $(h g)^{2}=-1$, then

$$
\psi_{1}=\left(Q, d_{-}^{\nabla}\right):\left(R_{+}^{k_{1}+3} \oplus R_{-}^{k_{2}+2}\right) \oplus V_{1} \rightarrow\left(R_{+}^{k_{1}} \oplus R_{-}^{k_{2}}\right) \oplus W_{1} .
$$

Here the sign \pm means the \pm 1 eigenspace of $h g$. If $(h g)^{2}=1$, then

$$
\begin{gathered}
\nu\left(\mathscr{B}^{H}, \mathscr{B}\right)_{x}=R_{-}^{k_{2}+4}+\left(V_{1}\right)_{-}, \\
\nu(Z, F)_{x}=\Omega_{-}^{2}\left(\mathscr{G}_{E}\right)=R^{k} \oplus W_{1} \\
=\left(R_{+}^{k_{1}} \oplus\left(W_{1}\right)_{+}\right) \oplus\left(R_{-}^{k_{2}} \oplus\left(W_{1}\right)_{-}\right), \\
\nu(Z, F)_{H, x}=\left(R_{-}^{k_{2}} \oplus\left(W_{1}\right)_{-}\right),
\end{gathered}
$$

where $\left(V_{1}\right.$ and $\left.W_{1}\right),\left(V_{1+}\right.$ and $\left.W_{1+}\right)$, and $\left(V_{-}\right.$and $\left.V_{1-}\right)$ are $G$-equivariant Hilbert space isomorphisms by $d_{-}^{\nabla}$. Thus the fiber

$$
\begin{aligned}
V(H)_{x} & =\operatorname{Hom}_{H}^{s}\left(\nu\left(\mathscr{B}^{H}, \mathscr{B}\right)_{x}, \nu(X, F)_{H, x}\right) \\
& =\operatorname{Hom}^{s}\left(R_{-}^{k_{2}+4} \oplus\left(V_{1}\right)_{-}, R_{-}^{k_{2}} \oplus\left(W_{1}\right)_{-}\right) \\
& = \begin{cases}\text {contractible } & \text { if } \operatorname{dim} V_{1-}=\infty, \\
V_{k_{2}+4, k_{2}} & \text { if } \operatorname{dim} V_{1-}<\infty .\end{cases}
\end{aligned}
$$

If $(h g)^{2}=-1$, then

$$
\nu\left(\mathscr{B}^{H}, \mathscr{B}\right)_{x}=R_{-}^{k_{2}+2} \oplus\left(V_{1}\right)_{-}, \quad \nu(Z, F)_{H, x}=R_{-}^{k_{2}} \oplus\left(W_{1}\right)_{-} .
$$

(6.3) The fiber $V(H)_{x}=\operatorname{Hom}^{s}\left(R_{-}^{k_{2}+2}, R_{-}^{k_{2}}\right)$ is the Stiefel manifold $V_{k_{2}+2, k_{2}}$ which consists of all $k_{2}$-frames in $R^{k_{2}+2}$. 
(6.4) The Stiefel manifold $V_{n, k}$ is arcwise-connected and

$$
\begin{aligned}
& \pi_{i}\left(V_{n, k}\right)=0 \text { if } i<n-k, \\
& \pi_{n-k}\left(V_{n, k}\right)= \begin{cases}\text { infinite cyclic group } & \text { if } n-k \text { is even or } k=1, \\
Z_{2} & \text { if } n-k \text { is odd and } k>1 .\end{cases}
\end{aligned}
$$

By (6.2), (6.3), and (6.4) we have

(6.5) In the bundle $V(H) \rightarrow X$, the fiber has the fundamental groups as follows:

$$
\pi_{i}\left(V(H)_{x}\right)= \begin{cases}Z & \text { if }(h g)^{2}=-1 \text { and } i=2, \\ 0 & \text { if }(h g)^{2}=+1 \text { and } i \leq 3\end{cases}
$$

where $h(x)=h(\nabla)=g(\nabla)$.

Moreover if $(h g)^{2}=1$, then the obstructions cohomology class $O_{n}\left(\psi_{1}\right) \in$ $H^{n}\left(X, X_{0}: \pi_{n-1}(V(H))\right) \equiv 0$ for all $n$.

However the compact set $X \subset \mathscr{M}_{1}^{\hat{H}}=\mathscr{M}_{1}^{\wedge G} \subset \mathscr{M}_{1}=5$. This is incorrect because $\mathscr{M}_{\hat{1}}$ may not be a manifold. By Corollary $3.11 \mathscr{M}_{1}{ }^{\widehat{G}}$ is a disjoint union of 1-dimensional manifold components and 3-dimensional manifold components which correspond by $h(\nabla)=g(\nabla),(h g)^{2}=1$, or $(h g)^{2}=-1$ respectively. Thus $X=\bigcup_{i} x_{i}^{1} \bigcup_{i} X_{i}^{3}$ where $\operatorname{dim} X_{i}^{1}=1$ and $\operatorname{dim} X_{i}^{3}=3$. If $h(\nabla)=g(\nabla),(h g)^{2}=-1$, then the obstruction cohomology classes $\Theta_{3, i}\left(\psi_{1}\right) \in$ $H^{3}\left(X_{i}^{3}, X_{i 0}^{3} ; Z\right)$, where $X_{i 0}^{3}=X_{i}^{3} \cap X_{0}$.

Theorem 6.6. (i) To perturb $\psi: \mathscr{B} \rightarrow \mathscr{C} \times_{\mathscr{G}} \Omega_{-}^{2}\left(\mathscr{G}_{E}\right)$ to be $G$-transversal throughout a neighborhood of $\mathscr{M}^{G}$ there are the obstructions $\Theta_{3}\left(\psi_{1}\right) \in H^{3}\left(X, X_{0} ; Z\right)$.

(ii) If the obstructions $\Theta_{3}\left(\psi_{1}\right)=0$, then the G-section $\psi$ has a smooth compact $G$-perturbation $R_{-}+\sigma$ of the self-dual Yang-Mills equations which is transversal to the zero section throughout a small neighborhood of $\mathscr{M}_{1}^{G}$.

\section{Perturbation on the free part of}

In [7], it is shown that there is a $G$-invariant metric on $M$ such that the moduli space $\mathscr{M}$ is a manifold in a $G$-neighborhood of the fixed point set $\mathscr{M}^{G}$. Suppose that the obstruction cohomology class $\Theta_{3}(\phi)=0$ and that the map $\mathscr{B} \stackrel{\psi}{\longrightarrow} \mathscr{F}=\mathscr{C} \times_{\mathscr{G}} \Omega_{-}^{2}\left(\mathscr{G}_{E}\right)$ is the Fredholm $G$-map which is transverse to the zero section throughout a $G$-neighborhood $N\left(\mathscr{M}^{G}\right)$ of the fixed point set $\mathscr{M}^{G}$. Let $Y=\mathscr{M} \backslash\left\{N\left(\mathscr{M}^{G}\right) \cup\right.$ End of $\left.\mathscr{M}\right\}$. Then $Y$ is a compact subset of $\mathscr{M}$ and $\mathscr{M} \backslash Y$ is a smooth 5-dimensional manifold with some singular points. For each $\nabla \in Y$ we can choose a local coordinate $\Theta_{\nabla \cdot \varepsilon}=\left\{A \in \Omega_{3}^{1}\left(\mathscr{G}_{E}\right) \mid \delta^{\nabla} A=\right.$ $\left.0,\|A\|_{3}<\varepsilon\right\}$ if $\nabla$ is irreducible, otherwise $\Theta_{\nabla \cdot \varepsilon} / U(1)$ is a local coordinate chart at $\nabla$ such that $h\left(\Theta_{\nabla \cdot \varepsilon}\right) \cap \Theta_{\nabla \cdot \varepsilon}=\varnothing$ where $h$ is the generator of $G=Z_{2}$. To see the local structure of the map $\psi: \mathscr{B}=\mathscr{C} / \mathfrak{G} \rightarrow \mathscr{F}=\mathscr{C} \times, \Omega_{-}^{2}\left(\mathscr{G}_{E}\right)$ and 
its interaction with the $G$-action, let us consider the following diagram:

$$
\begin{aligned}
& V_{0}+V_{1}=V \supset \Theta_{\nabla \cdot \varepsilon} \quad \frac{\psi}{\left(Q_{1}, h_{1}\right)} \Omega_{-}^{2}\left(\mathscr{G}_{E}\right)_{\nabla}=W_{0} \oplus W_{1} \\
& \downarrow h \\
& \downarrow h \\
& V_{0}^{\prime}+V_{1}^{\prime}=h_{*}(V) \supset h\left(\Theta_{\nabla \cdot \varepsilon}\right) \underset{\left(Q_{2}, h_{2}\right)}{\longrightarrow} \Omega_{-}^{2}\left(\mathscr{G}_{E}\right)_{h(\nabla)}=W_{0}^{\prime} \oplus W_{1}^{\prime}
\end{aligned}
$$

Since $\psi$ is a $G$-map, the above diagram commutes. Since $\psi$ is a Fredholm map with index $5, \psi$ becomes locally $\psi=(Q, L): V_{0} \oplus V_{1} \rightarrow W_{0} \oplus W_{1}$ by some $G$-equivariant diffeomorphism where $Q, L$ are $G$-maps, $\left.d \psi\right|_{v,}=$ $\left.L\right|_{v_{1}}: V_{1} \rightarrow W_{1}$ is a Hilbert space isomorphism, and $\left.Q\right|_{v_{0}}: v_{0}=R^{k+5}\left(C^{k+3}\right) \rightarrow$ $W_{0} \equiv R^{k}\left(C^{k}\right)$ is also a $G$-map with $d Q=0$ (if $\nabla$ is reducible) (cf. [14, Lemma 4.7]). Since $h$ is diffeomorphic, we can locally identify $h$ with its differential at the origin $\nabla$. Let $d \psi_{\nabla}=L_{1}$ and $d \psi_{h(\nabla)}=L_{2}$. Since $\psi h=h \psi$ we get $L_{2} h=h L_{1}$. Since $V_{0}$ is the kernel of $L_{1}$ and $L_{1}: V_{1} \rightarrow W_{1}$ is an isomorphism,

$$
\begin{aligned}
& L_{2}\left[h V_{0}\right]=h\left[L_{1} V_{0}\right]=h(0)=0, \quad \text { so } h\left(V_{0}\right) \in \operatorname{Ker} L_{2}, \\
& L_{2}\left[h V_{1}\right]=h\left[L_{1} V_{1}\right]=h\left(W_{1}\right), \quad \text { so } L_{2}: h\left(V_{1}\right) \stackrel{\text { iso }}{\cong} h\left(W_{1}\right) .
\end{aligned}
$$

Thus we have the canonical splitting $\psi=\left(Q_{2}, L_{2}\right): h\left(V_{0}\right) \oplus h\left(V_{1}\right) \rightarrow h\left(W_{0}\right) \oplus$ $h\left(W_{1}\right)$ at a neighborhood of $h(\nabla)$.

(7.1) For each $\nabla \in Y$ the generator $h \in Z_{2}$ preserves the local splitting of the Fredholm map $\psi: \mathscr{B} \rightarrow \mathscr{F}$.

With these preliminaries let us perturb $\psi$ on $Y \subset \mathscr{M}$. Suppose that $\nabla \in Y$ is reducible. Then $h(\nabla)$ is also reducible. We may choose a small neighborhood $\Theta_{\nabla, \varepsilon}$ of $\nabla$ with $\Theta_{\nabla, \varepsilon} \cap h\left(\Theta_{\nabla, \varepsilon}\right)=\varnothing$. There is a perturbation $\sigma: \Theta_{\nabla, \varepsilon} \rightarrow \Omega_{-}^{2}\left(\mathscr{G}_{E}\right)$ such that a new section $\psi_{1}=\psi+\sigma: \mathscr{B} \rightarrow \mathscr{F}$ is transverse to the zero section throughout $\Theta_{\nabla \cdot \varepsilon}$. Define a perturbation on $h\left(\Theta_{\nabla \cdot \varepsilon}\right)$ by $\sigma(h(A))=h \sigma(A)$. We have a $G$-equivariant section $\psi_{1}=\psi_{2}+\sigma: \mathscr{B} \rightarrow \mathscr{F}$ which is transverse to the zero section throughout $\Theta_{\nabla \cdot \varepsilon} \cup h\left(\Theta_{\nabla \cdot \varepsilon}\right)$. Since this is a compact perturbation, if we mod out the zero set at $\nabla$ by $U(1)$, then this reducible connection has a neighborhood which is a cone on $\mathbf{C} P^{2}$. Adding such a perturbation at each reducible connection in $Y$, we have a section $\psi_{3}: \mathscr{B} \rightarrow \mathscr{F}$ which is transverse to the zero section near the reducible connections in $\mathscr{M}_{1}=\left\{\nabla \in \mathscr{B} \mid \psi_{3}(\nabla)=0\right\}$. Thus we have

(7.2) Suppose that $\nabla \in Y$ is reducible. Then there is a $G$-equivariant compact perturbation of $\psi$ so that $\mathscr{M}_{1}$ has a cone-neighborhood on $\mathbf{C} P^{2}$ at $\nabla$.

Let $Y_{1}=\mathscr{M}_{1}-\left\{N\left(\mathscr{M}^{G}\right) \cup\right.$ End of $\mathscr{M} \cup$ [cones on $\mathbf{C} P^{2}$ at reducible self-dual connections]\}. 
Let $\nabla \in Y_{1}$ be irreducible, where $Y_{1}$ is compact. The Fredholm map $\psi_{3}$ locally splits as $\psi_{3}=(Q, L): \Theta_{v \cdot \varepsilon} \subset V=V_{0} \oplus V_{1} \rightarrow \Omega_{-}^{2}\left(\mathscr{G}_{E}\right)=W_{0} \oplus W_{1}$, where $L=d \psi_{3}: V_{1} \rightarrow W_{1}$ is a Hilbert isomorphism, $V_{0}=R^{k+5}, W_{0}=R^{k}$, and where each map is a $G$-map and each space is a $G$-space.

Choose a smooth cutoff function $\rho \in C_{0}\left(\Theta_{\nabla \cdot \varepsilon}\right)$ and consider the family of perturbations $\sigma_{W} \equiv \rho \cdot w: \Theta_{\nabla \cdot \varepsilon} \rightarrow R_{\nabla}^{k} \subset \Omega_{-}^{2}\left(\mathscr{G}_{E}\right)$ for each $w \in R^{k}=W_{0}$. As above, extend the perturbation by $h\left(\sigma_{w} A\right)=\sigma_{h w}(h A)$ on $h\left(\Theta_{\nabla \cdot \varepsilon}\right) \stackrel{\sigma_{h(w)}}{\longrightarrow}$ $R_{h(\nabla)}^{k} \subset \Omega_{-}^{2}\left(\mathscr{G}_{E}\right)_{h(\nabla)}$ for each $h W \in R_{h(\nabla)}^{k}$ (cf. Lemma 6.1). By considering the $G$-map $Q_{\nabla} \mid R_{\nabla}^{k+5} \rightarrow R_{\nabla}^{k}$ we have the following immediate consequence.

Lemma 7.3. $w \in R_{\nabla}^{k}$ is a regular value of $Q_{\nabla}: R_{\nabla}^{k+5} \rightarrow R_{\nabla}^{k}$ if and only if $h(w) \in R_{h(\nabla)}^{k}$ is a regular value of $Q_{h(\nabla)}: R_{h(\nabla)}^{k+4} \rightarrow R_{h(\nabla)}^{k}$.

We can cover the compact set $Y_{1}$ with the supports of a finite number of such perturbations. We get a family of perturbations $\psi_{w}=\psi_{3}+\sigma_{w}+\sigma_{h w_{1}}+$ $\cdots+\sigma_{w_{n}}+\sigma_{h w_{n}}$ for each $w=\left(w_{1}, \ldots, w_{n}\right) \in R^{k_{1}} \times \cdots \times R^{k_{n}} \equiv R^{m}$.

We may assume that the support of the perturbation lies in a small neighborhood of $Y_{1}$. Let a smooth mapping $\tilde{\psi}: \mathscr{B} \times B^{m}(\eta) \rightarrow \mathscr{F}$ be defined by $\tilde{\psi}(x, w)=\psi_{w}(x)$, where $B^{m}(\eta)=\left\{w \in R^{m}:\|w\|<\eta\right\}$.

(7.4) For small $\eta>0$, this mapping $\tilde{\psi}: \mathscr{B} \times B^{m}(\eta) \rightarrow \mathscr{F}$ is transversal to the zero section $Z \subset \mathscr{F}$.

Proof. Suppose that $(x, w) \in \mathscr{B} \times B^{m}(\eta)$ with $\tilde{\psi}(x, w)=0$.

(i) If $x \notin$ support of $\rho_{i}$ for all $i$, then $\tilde{\psi}(x, w)=\psi_{3}(x)=0$, and $\tilde{\psi}$ is already transversal by our construction.

(ii) If $x \in$ support of $\rho_{i}$ for some $i$, then $x \in \operatorname{supp} \rho_{i} \subset \Theta_{\nabla_{i} \cdot \varepsilon}$. Write $\tilde{\psi}(x, w)=\psi_{3}(x)+\sigma\left(x, \bar{w}_{i}\right)+\rho_{i}(x) w_{i}$. Then $\bar{w}_{i}=\left(w_{1} \cdots w_{i}^{-} \cdots w_{n}\right)$, where $\sigma\left(x, \bar{w}_{i}\right)=\sum_{i \neq j} \sigma_{w_{i}}(x)$ is uniformly $c^{1}$-small.

This is guaranteed by choosing $\eta$ small after covering with a finite number of coordinate charts. Note that $d\left(\psi_{3}+\sigma\right)_{x}: V=V_{0} \oplus V_{1} \rightarrow W=W_{0} \oplus W_{1}$ will still be transverse to $W_{1}$. Also $\sigma_{i}$ is the map

$$
R^{k+5} \times R^{k_{i}} \stackrel{\rho_{i} \times \mathrm{id}}{\longrightarrow} R \times R^{k_{i}} \stackrel{\text { scalar multi }}{\longrightarrow} R^{k_{i}},
$$

which has a surjective differential. Namely the $w_{i}$-spaces are carried onto $w_{0}$. Hence the total differential is surjective, i.e., $\psi \pitchfork Z$.

By Sard's theorem for families, the map $\psi_{w}=\psi_{3}+\sigma_{w_{1}}+\sigma_{h w_{1}}+\cdots+\sigma_{w_{n}}+\sigma_{h w_{n}}$ is transversal to the zero section for almost all $w \in B^{m}(\eta)$.

Lemma 7.5. $\psi_{u}: \mathscr{B} \rightarrow \mathscr{F}$ is a G-map. 
Proof. If $A \notin \operatorname{supp} \rho_{i}$ for all $i$, then $h(A) \notin \operatorname{supp} \rho_{i}$ for all $i$ and $\psi_{w}(h A)=$ $\psi_{3}(h A)=h \psi_{3}(A)=h \psi_{w}(A)$. If $A \in \operatorname{supp} \rho_{i}$ for some $i$, then $A \in \operatorname{supp} \rho_{i} \subset$ $\Theta_{\nabla \cdot \varepsilon}$ and $h(A) \in h\left(\Theta_{\nabla, \varepsilon}\right)$. By our construction $\Theta_{\nabla \cdot \varepsilon} \cap h\left(\Theta_{\nabla \cdot \varepsilon}\right)=\varnothing$ and

$$
\begin{aligned}
\psi_{w}(h A) & =\psi_{3}(h A)+\sigma_{w_{1}}(h A)+\sigma_{h w_{1}}(h A)+\cdots+\sigma_{w_{n}}(h A)+\sigma_{h w_{n}}(h A) \\
& =h \psi_{3}(A)+h \sigma_{h w_{1}}(A)+h \sigma_{w_{1}}(A)+\cdots+h \sigma_{h w_{n}}(A)+h \sigma_{w_{n}}(A) \\
& =h\left[\psi_{3}(A)+\sigma_{h w_{1}}(A)+\sigma_{w_{1}}(A)+\cdots+\sigma_{h w_{n}}(A)+\sigma_{w_{n}}(A)\right] \\
& =h \psi_{w}(A) .
\end{aligned}
$$

Theorem 7.6. There is a compact G-equivariant perturbation $\psi_{4}=\psi_{3}+\sigma_{2}$ of the perturbed self-dual equation $\psi_{3}=R_{-}+\sigma_{1}$ so that the new moduli space $\mathscr{M}_{2}=\left\{\nabla \in \mathscr{B}: \psi_{4}(\nabla)=0\right\}$ is a smooth 5-dimensional G-manifold with $\lambda$ singularities each of which has a neighborhood diffeomorphic to the cone on $\mathbf{C} P^{2}$ except the cone point, where $\lambda=\operatorname{rank} H^{2}(M ; Z)$.

If the obstruction cohomology classes $\Theta_{3}(\psi)$ vanish, then we have a smooth $G$-manifold $\mathscr{M}$ of dimension 5 with $\lambda$-singular points each of which has a cone neighborhood on $\mathbf{C} P^{2}$, where $\lambda=\operatorname{rank} H^{2}(M ; Z)$.

Acknowledgment. The author wishes to thank Professors Melvin Rothenberg and Karen Uhlenbeck for their advice, help, and encouragement during the course of this work.

\section{REFERENCES}

1. M. F. Atiyah, N. Hitchin, and I. Singer, Self duality in four-dimensional Riemannian geometry, Proc. Roy. Soc. London Ser. A 362 (1978), 425-461.

2. M. F. Atiyah and G. B. Segal, The index of elliptic operators. II, Ann. of Math. (2) 87 (1968), 531-545.

3. M. F. Atiyah and I. Singer, The index of elliptic operators. III, Ann. of Math. (2) 87 (1968), 546-604.

4. _ The index of elliptic operators. IV, V, Ann. of Math. (2) 93 (1971), 119-149.

5. J. P. Bourguignon and H. B. Lawson, Jr., Stability and isolation phenomena for Yang-Mills theory, Comm. Math. Phys. 79 (1982), 189-230.

6. Yang-Mills theory, its physical origins and differential geometric aspects, Ann. of Math. Studies, no. 102, Princeton Univ. Press, Princeton, N.J., 1982, pp. 395-421.

7. Y. S. Cho, Finite group actions on the moduli space of self-dual connections. II, Michigan Math. J. 37 (1990).

8. J. Cheeger and D. Ebin, Comparison theorems in Riemannian geometry, North-Holland, Amsterdam, 1975.

9. S. Donaldson, An application of gauge theory to four-manifold theory, J. Differential Geom. 18 (1983), 279-315.

10. _ Connections, cohomology and the intersection forms of 4-manifolds: J. Differential Geom. 24 (1986), 275-341.

11. R. Fintushel and R. Stern, SO(3)-connections of topology of 4-manifold, J. Differential Geom. 20 (1984), 523-539.

12. __, Pseudofree orbifolds, Ann. of Math. (2) 122 (1985), 335-364. 
13. __ Definite 4-manifold, J. Differential Geom. 28 (1988), 133-142.

14. D. Freed and K. Uhlenbeck, Instantons and four-manifolds, Math. Sci. Res. Inst. Publ., vol. 1, Springer-Verlag, New York, 1984.

15. M. Freedman, The topology of four dimensional manifolds, J. Differential Geom. 17 (1983), $357-454$.

16. I. Hambleton and R. Lee, Finite group actions on $\mathbf{C} P^{2}$, preprint.

17. F. Hirzebruch, Topological methods in algebraic geometry, 3rd ed., Springer-Verlag, New York, 1978.

18. N. H. Kuiper, The homotopy type of the unitary group of Hilbert space, Topology 3 (1965), 19-30.

19. B. Lawson, Jr., The theory of gauge fields in four dimensions, CBMS Regional Conf. Ser. Math., no. 58, Amer. Math. Soc., Providence, R.I., 1985.

20. J. Milnor and J. Stasheff, Characteristic classes, Princeton Univ. Press, Princeton, N.J., 1974.

21. T. Parker, Gauge theories on four dimensional Riemannian manifolds, Comm. Math. Phys. 85 (1982), 1-40.

22. T. Petrie, Pseudo equivalence of G-manifolds, Proc. Sympos. Pure Math., vol. 32, Amer. Math. Soc., Providence, R.I., 1978, pp. 169-210.

23. T. Petrie and J. Randall, Transformation groups on manifolds, Pure Appl. Math. 82 (1984).

24. P. Shanahan, The Atiyah-Singer index theorem, Lecture Notes in Math., vol 638, SpringerVerlag, 1970.

25. I. Singer, Some remarks on the Gribov ambiguity, Comm. Math. Phys. 60 (1978), 7-12.

26. I. Singer and J. Thorpe, The curvature of 4-dimensional Einstein spaces, Global Analysis, Princeton Univ. Press, Princeton, N.J., 1969, pp. 335-365.

27. S. Smale, An infinite dimensional version of Sard's theorem, Amer. J. Math. 87 (1968), 861-866.

28. N. Steenrod, The topology of fiber bundles, Princeton Univ. Press, Princeton, N.J., 1951.

29. R. Stern, Instantons and the topology of 4-manifolds, Math. Intelligencer 5 (1983), 39-44.

30. C. Taubes, Self-dual connections on non-self-dual 4-manifolds, J. Differential Geom. 17 (1982), 139-170.

Department of Mathematics, Brandeis University, Waltham, Massachusetts 02154

Current address: Department of Mathematics, College of Natural Sciences, Ewha Womans University, Seoul 120-750, Korea 